\title{
QUASI-MONTE CARLO METHODS FOR HIGH-DIMENSIONAL INTEGRATION: THE STANDARD (WEIGHTED HILBERT SPACE) SETTING AND BEYOND
}

\author{
F. Y. KUO ${ }^{1}$, CH. SCHWAB ${ }^{2}$ and I. H. SLOAN ${ }^{\bowtie 1, *}$
}

(Received 30 December, 2011; revised 3 February, 2012)

\begin{abstract}
This paper is a contemporary review of quasi-Monte Carlo (QMC) methods, that is, equal-weight rules for the approximate evaluation of high-dimensional integrals over the unit cube $[0,1]^{s}$. It first introduces the by-now standard setting of weighted Hilbert spaces of functions with square-integrable mixed first derivatives, and then indicates alternative settings, such as non-Hilbert spaces, that can sometimes be more suitable. Original contributions include the extension of the fast component-by-component (CBC) construction of lattice rules that achieve the optimal convergence order (a rate of almost $1 / N$, where $N$ is the number of points, independently of dimension) to so-called "product and order dependent" (POD) weights, as seen in some recent applications. Although the paper has a strong focus on lattice rules, the function space settings are applicable to all QMC methods. Furthermore, the error analysis and construction of lattice rules can be adapted to polynomial lattice rules from the family of digital nets.
\end{abstract}

\begin{abstract}
2010 Mathematics subject classification: primary 65D30; secondary 65D32.
Keywords and phrases: quasi-Monte Carlo methods, QMC, high-dimensional integration, weighted spaces, reproducing kernel Hilbert spaces, Banach space settings, worst-case error, discrepancy, weighted Koksma-Hlawka inequality, lattice rules, lowdiscrepancy sequences, fast $\mathrm{CBC}$ construction, $\mathrm{POD}$ weights.
\end{abstract}

\section{Introduction and some QMC basics}

Quasi-Monte Carlo (QMC) methods are deterministic methods for high-dimensional integration which aim to outperform the classical Monte Carlo method. In the past fifteen years great progress has been made, often in the setting of "worst-case" errors in a "weighted” Hilbert space, as introduced originally by Sloan and Woźniakowski [53].

\footnotetext{
* This is a contribution to the series of invited papers by past ANZIAM medallists (Editorial, issue 52(1)). Ian Sloan was awarded the 1997 ANZIAM medal.

${ }^{1}$ School of Mathematics and Statistics, University of New South Wales, Sydney NSW 2052, Australia; e-mail: f.kuo@unsw.edu.au,i.sloan@unsw.edu.au.

${ }^{2}$ Seminar for Applied Mathematics, ETH Zürich, ETH Zentrum, HG G57.1, CH8092 Zürich, Switzerland; e-mail: christoph.schwab@sam.math.ethz.ch.

(c) Australian Mathematical Society 2012, Serial-fee code 1446-1811/2012 \$16.00
} 
This we call the "standard setting". The first aim of this paper is to explain this standard setting, but much of the paper is concerned with extensions that go beyond it. These extensions include non-Hilbert space settings, infinite-dimensional problems, and nonstandard choices of "weights".

This paper is in part motivated by a recent application [31] by the present authors of QMC methods to the computation of expected values of functionals of the solutions to second-order partial differential equations with random coefficients. While we do not review our other paper [31] here, that work encouraged us to look beyond the standard setting. For instance, the dimensionality in that problem is not just high but truly infinite, and the weights that we were led to use are certainly nonstandard (see Section 1.4). Also, although that paper in its final form is concerned with the particular kind of QMC methods known as lattice rules, and stays within a Hilbert space setting, in the course of the research we considered many other possibilities, and still believe that other QMC methods and non-Hilbert space settings offer exciting possibilities for this application as well as others.

For the majority of this article we focus on the integration problem over the $s$-dimensional unit cube,

$$
I_{s}(F)=\int_{[0,1]^{s}} F(\boldsymbol{y}) d \boldsymbol{y},
$$

with the dimensionality $s$ being large but finite. Here $F$ is a function that is Lebesgue integrable over $[0,1]^{s}$, with some extra smoothness so that, in particular, evaluations of $F$ at points in $[0,1]^{s}$ are well defined. An $N$-point QMC approximation to the integral (1.1) is an equal-weight quadrature rule of the form

$$
Q_{s, N}(F)=\frac{1}{N} \sum_{i=1}^{N} F\left(y^{(i)}\right),
$$

with a well-chosen set of points $\mathcal{P}=\left\{\boldsymbol{y}^{(1)}, \ldots, \boldsymbol{y}^{(N)}\right\} \subset[0,1]^{s}$.

What is so good about QMC rules? Why not use, for example, a product of onedimensional Gauss rules? The short answer is that while product Gauss rules could work well, and indeed might be the recommended option when $s$ is small and $F$ is sufficiently smooth (see, for example, the book by Stroud [58]), every product rule is infeasible when $s$ is large. Suppose, for example, that $s=100$. Then even a product of 2 -point rules will require $2^{100}$ points, a number that is certain to be beyond our reach for many lifetimes. This is a manifestation of the famous curse of dimensionality [2].

But even if product rules are excluded, why do we restrict ourselves to equal weights? The QMC rules integrate constants exactly, but in general fail to integrate exactly all polynomials of higher degree. Perhaps if we allowed the weights to be unequal, we could integrate exactly at least some higher-degree polynomials? Once again, the curse of dimensionality is against us: for example, if we want to integrate exactly all multilinear polynomials (that is, all functions that are linear with respect to each component of $\boldsymbol{y}$ ), then we must satisfy $2^{s}$ independent conditions. But perhaps the real reason for using equal weights is that this is the simplest choice to analyse. 
Error bounds for QMC methods generally take the form of an inequality

$$
\left|I_{s}(F)-Q_{s, N}(F)\right| \leq D(\mathcal{P}) V(F),
$$

in which the first factor, a discrepancy, is independent of $F$ and is a measure of the quality of the point set $\mathcal{P}$, while the second factor is independent of the point set and is a measure of the difficulty of the integrand $F$. The prototype of such inequalities is the Koksma-Hlawka inequality, which we review briefly in Section 1.3. An inequality of this form has the nice feature that, for a given function $F$, the error bound will be reduced to the extent that we can reduce the discrepancy of the point set $\mathcal{P}$. (Note, however, that the error itself may not be reduced: the guarantee only pertains to the error bound.)

Much of the literature on QMC methods talks about worst-case error, not discrepancy. To avoid later pain, it may help to realize that these terms usually refer to the same thing. To define worst-case error, suppose that the integrand $F$ is constrained to lie in some Banach space $\mathcal{W}$ with norm $\|F\|_{W}$. (The choice of function space is limited by the natural requirement that pointwise function values make sense. We give many examples later. For now, think of $s=1$, and take either $\mathcal{W}=C[0,1]$ or $\mathcal{W}=H^{1}[0,1]$, the space of square-integrable functions on $[0,1]$, with some appropriate norm in $H^{1}[0,1]$; see Section 1.1 for an example.) Then the worstcase error is defined by

$$
e^{\text {wor }}(\mathcal{P} ; \mathcal{W}):=\sup \left\{\left|I_{s}(F)-Q_{s, N}(F)\right|:\|F\|_{\mathcal{W}} \leq 1\right\} .
$$

Since the error depends linearly on $\|F\|_{\mathcal{W}}$, it follows that for all $F \in \mathcal{W}$,

$$
\left|I_{s}(F)-Q_{s, N}(F)\right| \leq e^{\text {wor }}(\mathcal{P}, \mathcal{W})\|F\|_{\mathcal{W}},
$$

which is of the same form as (1.3), with the discrepancy replaced by the worst-case error and $V(F)$ replaced by the norm $\|F\|_{w}$. Often $V(F)$ is a seminorm rather than a norm, but the distinction is usually unimportant. (We could equally well use a seminorm in the definition of the worst-case error, but it would be nonstandard to do so.) If $D(\mathcal{P})$ is the smallest possible constant in (1.3) and $V(F)$ is a norm or seminorm, then $D(\mathcal{P})$ is indeed a worst-case error.

1.1. The standard setting for QMC In the standard setting, introduced by Sloan and Woźniakowski [53], the quantity $V(F)$ is a norm in a Hilbert space $\mathcal{H}_{s, \gamma}$ of functions $F$ that enjoy some smoothness and whose mixed first derivatives are all square-integrable over $[0,1]^{s}$; these norms also incorporate weights, which are positive numbers $\gamma_{1}, \gamma_{2}, \ldots, \gamma_{s}$ designed to quantify the different degrees of difficulty associated with the different components of $\boldsymbol{y}$ in (1.1). Here we always assume that

$$
\gamma_{1} \geq \gamma_{2} \geq \cdots \geq \gamma_{s}>0
$$

corresponding to the idea that the first component of $\boldsymbol{y}$ is the hardest one for this integrand, the second component the next hardest, and so on. 
For $s=1$, the (real) space $\mathcal{H}_{1, \gamma_{1}}$ consists of absolutely continuous functions whose first derivatives are integrable. The inner product in $\mathcal{H}_{1, \gamma_{1}}$ is

$$
\langle F, G\rangle_{1, \gamma_{1}}:=F(1) G(1)+\frac{1}{\gamma_{1}} \int_{0}^{1} F^{\prime}(y) G^{\prime}(y) d y, \quad F, G \in \mathcal{H}_{1, \gamma_{1}},
$$

and thus the corresponding norm squared is

$$
\|F\|_{1, \gamma_{1}}^{2}=|F(1)|^{2}+\frac{1}{\gamma_{1}} \int_{0}^{1}\left|F^{\prime}(y)\right|^{2} d y, \quad F \in \mathcal{H}_{1, \gamma_{1}} .
$$

For $s=2, \mathcal{H}_{2, \gamma}$ is the tensor product of $\mathcal{H}_{1, \gamma_{1}}$ and $\mathcal{H}_{1, \gamma_{2}}$, and the norm squared in $\mathcal{H}_{2, \gamma}$ (we leave the inner product to be inferred from the norm) is defined by

$$
\begin{aligned}
\|F\|_{2, \gamma}^{2}:=|F(1,1)|^{2} & +\frac{1}{\gamma_{1}} \int_{0}^{1}\left|\frac{\partial F}{\partial y_{1}}\left(y_{1}, 1\right)\right|^{2} d y_{1}+\frac{1}{\gamma_{2}} \int_{0}^{1}\left|\frac{\partial F}{\partial y_{2}}\left(1, y_{2}\right)\right|^{2} d y_{2} \\
& +\frac{1}{\gamma_{1} \gamma_{2}} \int_{[0,1]^{2}}\left|\frac{\partial^{2} F\left(y_{1}, y_{2}\right)}{\partial y_{1} \partial y_{2}}\right|^{2} d y_{1} d y_{2} .
\end{aligned}
$$

Note that a small value of $\gamma_{2}$ forces the partial derivative $\partial F / \partial y_{2}$ to be small if $F$ is to stay within the unit ball in $\mathcal{H}_{2, \gamma}$. The point of this definition of $\|F\|_{2, \gamma}$ is that it matches the tensor product structure: for the special case of $F\left(y_{1}, y_{2}\right)=G\left(y_{1}\right) H\left(y_{2}\right)$, it is easily seen that $\|F\|_{2, \gamma}=\|G\|_{1, \gamma_{1}}\|H\|_{1, \gamma_{2}}$.

For general $s, \mathcal{H}_{s, \gamma}$ is the tensor product $\mathcal{H}_{1, \gamma_{1}} \otimes \mathcal{H}_{1, \gamma_{2}} \otimes \cdots \otimes \mathcal{H}_{1, \gamma_{s}}$, and we can write the norm squared in the more compact form

$$
\|F\|_{s, \gamma}^{2}:=\sum_{\mathfrak{u} \subseteq\{1: s\}} \gamma_{\mathfrak{u}}^{-1} \int_{[0,1]^{\mathfrak{u}}}\left|\frac{\partial^{|\mathfrak{u}|} F}{\partial \boldsymbol{y}_{\mathfrak{u}}}\left(\boldsymbol{y}_{\mathfrak{u}}, 1\right)\right|^{2} d \boldsymbol{y}_{\mathfrak{u}},
$$

where $\{1: s\}:=\{1, \ldots, s\}$ so that the sum is over all subsets $\mathfrak{u}$ of $\{1, \ldots, s\}$, and with $\boldsymbol{y}_{\mathfrak{u}}$ denoting the components $y_{j}$ of $\boldsymbol{y}$ with $j \in \mathfrak{u},\left(\boldsymbol{y}_{\mathfrak{u}}, 1\right)$ denoting the vector of length $s$ whose $j$ th component is $y_{j}$ if $j \in \mathfrak{u}$ and 1 if $j \notin \mathfrak{u}$, and

$$
\gamma_{\mathfrak{u}}:=\prod_{j \in \mathfrak{u}} \gamma_{j}
$$

Weights of this kind are nowadays referred to as "product" weights. It was assumed by Sloan and Woźniakowski [53] that $\gamma_{1}=1$, but this assumption is now considered to be unnecessary and too restrictive.

A short history of the standard setting is that the 1998 paper of Sloan and Woźniakowski [53] proved nonconstructively that for each $N \geq 1$ there exist QMC points $\left\{\boldsymbol{y}^{(1)}, \ldots, \boldsymbol{y}^{(N)}\right\} \subset[0,1]^{s}$ for which the worst-case error is bounded by $c / \sqrt{N}$, with $c$ independent of $s$, if and only if

$$
\sum_{j=1}^{\infty} \gamma_{j}<\infty
$$


Then, in 2000, Hickernell and Woźniakowski [21] proved (again nonconstructively) that the bound can be improved to $c_{\delta} / N^{1-\delta}$ for arbitrary $\delta>0$ under a stronger condition on the weights, most simply that

$$
\sum_{j=1}^{\infty} \gamma_{j}^{1 / 2}<\infty
$$

Sloan and Woźniakowski [54] then showed that there exists a QMC rule for which the worst-case error has a bound of the form $c_{\delta} / N^{1-\delta}$ even if the choice of QMC rule is restricted to the relatively small class of shifted lattice rules (see Section 1.2). The proof, like that in the earlier paper [53], uses an averaging argument (that there is at least one choice as good as the average) and is nonconstructive. In 2002, Sloan et al. [49] devised a component-by-component (CBC) construction of a shifted lattice rule that for $N$ prime achieves the $c / \sqrt{N}$ bound for the worst-case error in the standard setting, thereby achieving for prime $N$ a constructive proof of the result of Sloan and Woźniakowski [53]. The same authors also proposed a randomized version of the CBC construction [50], which was shown subsequently by Kuo [30] and Dick [5] to achieve the $c_{\delta} / N^{1-\delta}$ bound under the condition (1.10). Fast implementations of CBC constructions were introduced by Nuyens and Cools [42, 43], while modified algorithms for obtaining lattice rules that are extensible in $N$ were given by Cools et al. [4] and Dick et al. [7]. These made feasible the construction of explicit lattice rules that match the rates of convergence of the worst-case error in the existence results for all values of $s$ and $N$ that are likely to be of interest.

1.2. Lattice methods Much of this review focuses on an important family of QMC rules called shifted lattice rules. These take the form

$$
Q_{s, N}(F)=\frac{1}{N} \sum_{i=1}^{N} F\left(\left\{\frac{i z}{N}+\Delta\right\}\right),
$$

where $z \in \mathbb{N}^{s}$ is known as the generating vector, $\Delta \in[0,1]^{s}$ is the shift, and the braces $\{\boldsymbol{w}\}$ mean to take the fractional part of each component of the vector $\boldsymbol{w}$. Shifted lattice rules therefore require the specification of two vector quantities: an integer vector $z \in \mathbb{N}^{s}$ and a real number vector $\Delta \in[0,1]^{s}$.

Lattice rules without shift, namely those with $\boldsymbol{\Delta}=\mathbf{0}$, were originally introduced in a periodic function space setting (by Sloan and Joe [48], for example), but by now are seen to have an important role even for nonperiodic spaces. In the formula above, $\boldsymbol{\Delta}$ is deterministic. In contrast, a randomly shifted lattice rule takes the same form (1.11) where, as before, $z \in \mathbb{N}^{s}$ is a prescribed generating vector, but now each component of $\Delta \in[0,1]^{s}$ is a random variable that is uniformly and independently distributed in $[0,1]$. Randomly shifted lattice rules have come to play an important role, as foreshadowed in Section 1.1.

From a practical point of view, there are multiple advantages in using a randomized QMC rule: while enjoying nearly the optimal rate of convergence, a randomly shifted 
lattice rule is unbiased and provides a simple and practical error estimation, just like the Monte Carlo method. (Giles et al. [9, Section 5] give a brief explanation of how this error estimation is done in practice.)

The component-by-component (CBC) algorithm was invented for periodic spaces by Korobov [29] many years ago, and was rediscovered by Sloan and Reztsov [51]. The subsequent developments in the nonperiodic setting have already been mentioned in Section 1.1. In the CBC algorithm the integers $z_{1}, \ldots, z_{s}$ are chosen one at a time, in the natural order. Suppose that $z_{1}, \ldots, z_{s-1}$ are already determined. Then $z_{s}$ is determined by minimizing a certain quantity (the shift-averaged worst-case error) over the (at most $N-1$ ) possible values of $1 \leq z_{s} \leq N-1$ that are coprime with $N$. For further details about the CBC algorithm, see Section 4.

1.3. The classical setting and what goes wrong Here we briefly review the "classical" theory for obtaining bounds on the QMC error for nonperiodic functions, and explain the problem with its applicability in high dimensions. (For functions that are 1-periodic with respect to each of the $s$ variables, there is a theory based on Fourier analysis [36, 48]; we do not discuss the periodic case in this review.)

As foreshadowed in Section 1, the Koksma-Hlawka inequality plays an important role classically. This inequality takes the form

$$
\left|I_{s}(F)-Q_{s, N}(F)\right| \leq D^{*}(\mathcal{P}) V_{\mathrm{HK}}(F) .
$$

Here $V_{\mathrm{HK}}(F)$ is the variation of $F$ in the sense of Hardy and Krause (see the book by Niederreiter [36], for instance) and $D^{*}(\mathcal{P})$ is the (classical) star discrepancy of the QMC point set $\mathcal{P}=\left\{\boldsymbol{y}^{(1)}, \ldots, \boldsymbol{y}^{(N)}\right\}$, defined by

$$
D^{*}(\mathcal{P}):=\sup _{\boldsymbol{y} \in[0,1]^{s}}|\operatorname{discr} \mathcal{P}(\boldsymbol{y})|,
$$

where $\operatorname{discr}_{\mathcal{P}}(\cdot)$ is the local discrepancy function

$$
\operatorname{discr} \mathcal{P}(\boldsymbol{y}):=y_{1} y_{2} \cdots y_{s}-\frac{\left|\left\{i: \boldsymbol{y}^{(i)} \in[0, \boldsymbol{y})\right\}\right|}{N}, \quad \boldsymbol{y} \in[0,1]^{s},
$$

with $[0, \boldsymbol{y}):=\left[0, y_{1}\right) \times\left[0, y_{2}\right) \times \cdots \times\left[0, y_{s}\right)$. We discuss and prove more general versions of the Koksma-Hlawka inequality in Section 3.1.

The local discrepancy function is the difference between the volume of the rectangular region $[0, y)$ and the fraction of the QMC points that lie in the region. Intuitively, a small value of the star discrepancy means that the points are closer to being uniformly distributed.

By definition, an infinite sequence of points $\boldsymbol{y}^{(1)}, \boldsymbol{y}^{(2)}, \ldots$ in $[0,1]^{s}$ is a lowdiscrepancy sequence if for arbitrary $N \geq 1$ the star discrepancy of its first $N$ members satisfies

$$
D^{*}(\mathcal{P}) \leq C \frac{(\ln N)^{s}}{N}
$$

for some constant $C>0$ that is independent of $N$ but may depend on $s$. Examples of low-discrepancy sequences include Halton sequences [11], Sobol' 
sequences [55], Faure sequences [8], Niederreiter sequences [35, 36] and NiederreiterXing sequences [37]. Although a bound of the form (1.14) indicates an ultimate order of convergence theoretically higher than the classical Monte Carlo rate of $1 / \sqrt{N}$, that bound is unsatisfactory when the dimensionality is high because, for fixed $s,(\ln N)^{s} / N$ keeps growing with increasing $N$ until $N$ is exponentially large in $s$. In contrast to that somewhat negative observation is a remarkable result proved by Heinrich et al. [12], which states that there exists a sequence of QMC point sets for which the star discrepancy is of order $\sqrt{s / N}$ with an unknown constant, or alternatively $\sqrt{s \ln s \ln N / N}$ with an explicit constant. However, no one knows yet how to construct QMC points that satisfy a bound of this kind.

1.4. Why go beyond the standard setting? Within the standard setting we know (see Section 1.1) that, with the help of suitable weights, we can obtain close to order $1 / N$ for the worst-case error, with an implied constant independent of the dimension $s$, thus allowing QMC methods to be applicable to very high-dimensional problems. Why might one want to go beyond the standard setting? One argument concerns the choice of weights. While the theoretical results concerning weights in Section $1.1 \mathrm{might}$ be considered interesting, there have been few if any convincing prescriptions of the weights to use in any particular application: in most applications the choice of weights has been ad hoc. In contrast, in our other paper [31], the choice of weights is an essential ingredient in the analysis. Interestingly, the weights found there turn out to be not of the product form (1.8), but are rather of "product and order dependent" (POD) form, in general defined by

$$
\gamma_{\mathfrak{u}}=\Gamma_{\mid \mathfrak{u l}} \prod_{j \in \mathfrak{u}} \gamma_{j}
$$

where $|\mathfrak{u}|$ denotes the cardinality, or the order, of $\mathfrak{u}$. The multiplier $\Gamma_{|\mathfrak{u}|}$ in front of the product is a positive number that depends only on the order $|\mathfrak{u}|$ of the subset $\mathfrak{u}$, and hence is said to be order dependent. In the particular application in our other paper [31], the multiplier $\Gamma_{|\mathfrak{u}|}$ is found to be $\Gamma_{|\mathfrak{u}|}=(|\mathfrak{u}| !)^{v}$ for some positive number $v$ that is independent of $\mathfrak{u}$. Thus, for the problem considered there [31], confining ourselves to product weights as in (1.8) is no longer reasonable. In the next subsection we give another example, this time a simple one, in which it is again true that the best weights are POD weights. Fortunately, a general notion of weights (in which at the extreme all of the $2^{s}$ weights $\gamma_{\mathfrak{u}}$ in (1.7) are chosen independently) has already been proposed [52]. An efficient $\mathrm{CBC}$ construction is not available for all general weights but can be devised for the particular case of POD weights, which we show, as a new result, in Section 5.

Sometimes the Hilbert space aspect of the standard setting is a major restriction. This is often the case for problems whose natural setting is $\mathbb{R}^{s}$. For simplicity, let us think for a moment of functions in only one dimension, that is, $s=1$. Then an integral of the form

$$
\mathcal{I}_{1}(f):=\int_{-\infty}^{\infty} f(x) \rho(x) d x,
$$


where $\rho$ is a given probability density function on $\mathbb{R}$, can be reformulated as a problem on the unit cube via the transformation

$$
y=\Phi(x):=\int_{-\infty}^{x} \rho(t) d t,
$$

under which the integral $\mathcal{I}_{1}(f)$ becomes

$$
\mathcal{I}_{1}(f)=\int_{0}^{1} F(y) d y=I_{1}(F) \quad \text { with } F(y)=f\left(\Phi^{-1}(y)\right) .
$$

But the resulting integrand lies in our space $\mathcal{H}_{1, \gamma_{1}}$, with its norm defined by (1.6), if and only if $F^{\prime} \in L^{2}[0,1]$, where by the chain rule

$$
F^{\prime}(y)=\frac{f^{\prime}(x)}{\Phi^{\prime}(x)}=\frac{f^{\prime}\left(\Phi^{-1}(y)\right)}{\rho\left(\Phi^{-1}(y)\right)} .
$$

The problem comes from the requirement to square the denominator $\rho(x)$ : it is often the case that the resulting integral

$$
\int_{0}^{1}\left|F^{\prime}(y)\right|^{2} d y=\int_{0}^{1}\left|\frac{f^{\prime}\left(\Phi^{-1}(y)\right)}{\rho\left(\Phi^{-1}(y)\right)}\right|^{2} d y=\int_{-\infty}^{\infty} \frac{\left|f^{\prime}(x)\right|^{2}}{\rho(x)} d x
$$

diverges. In contrast, if the requirement were merely that $F^{\prime} \in L^{1}[0,1]$, then the demand would be merely

$$
\int_{0}^{1}\left|F^{\prime}(y)\right| d y=\int_{-\infty}^{\infty}\left|f^{\prime}(x)\right| d x<\infty,
$$

which is much more commonly satisfied. We discuss more general Banach space settings in Section 3 (this may be viewed as a "second course" on QMC).

There is another important family of QMC methods, called digital nets [36]. We do not discuss results relating to digital nets in this review. For recent developments on digital nets, including analysis in weighted spaces and constructions that achieve higher-order convergence (namely a convergence rate of order $N^{-\alpha}$ for $\alpha>1$ ), we refer the reader to the recent book by Dick and Pillichshammer [6].

1.5. A new kind of example Suppose that

$$
a(\boldsymbol{y})=a\left(y_{1}, \ldots, y_{s}\right):=1+\sum_{j=1}^{s} \frac{y_{j}^{\alpha}}{j^{2}}
$$

is a physical quantity that depends on $s$ independent random variables $y_{1}, \ldots, y_{s}$, each uniformly distributed over $[0,1]$, and that $0<\alpha \leq 1$. We note that

$$
1 \leq a(\boldsymbol{y}) \leq 1+\sum_{j=1}^{\infty} \frac{1}{j^{2}}=1+\frac{\pi^{2}}{6} \quad \text { for all } \boldsymbol{y} \in[0,1]^{s}, \text { for all } s \geq 1
$$


Our problem is to find the expected value of the reciprocal of $a$,

$$
F(y):=\frac{1}{a(y)} .
$$

Since the $y_{j}$ are independent and identically distributed uniformly over $[0,1]$, this expectation is given by

$$
I_{s}(F)=\int_{[0,1]^{s}} \frac{1}{a(\boldsymbol{y})} d \boldsymbol{y}=\int_{0}^{1} \ldots \int_{0}^{1} \frac{1}{a\left(y_{1}, \ldots, y_{s}\right)} d y_{1} \cdots d y_{s} .
$$

It is easily verified that $I_{s}(F)$ is well defined for $\alpha>0$.

The integrand $F(y)$ is a simplified model of an elliptic partial differential equation (PDE) with a random coefficient, as studied in our other paper [31]. Although the latter problem is beyond the scope of this paper, there, as here, the variables $y_{1}, y_{2}, \ldots$ are parameters in a "probability space". The big difference in that problem is the presence of another variable $\boldsymbol{x}$ in $\mathbb{R}^{2}$ or $\mathbb{R}^{3}$ corresponding to position in physical space, and that one has to solve an elliptic PDE with respect to the physical variable $\boldsymbol{x}$. If we instead write the definition (1.17) as an algebraic equation

$$
a(y) F(y)=1,
$$

then the present example has the flavour of the elliptic PDE considered in our other paper [31], while avoiding all PDE complications.

Suppose that we want to approximate $I_{S}(F)$ by a randomly shifted lattice rule; see (1.11). Then obviously we need to know a good choice for the integer vector $z$. In turn, if we want to generate a good choice of $\boldsymbol{z}$ by the CBC algorithm (see Sections 1.2 and 4), then we need to know a good choice of the weights $\gamma_{\mathfrak{u}}$ to steer the CBC algorithm.

The first step towards a rational choice of weights is to determine (or obtain a bound on) the norm $\|F\|_{s, \gamma}$. To this end, by direct differentiation of $F$ we find, with $d_{j}:=\alpha / j^{2}$,

$$
\begin{aligned}
\frac{\partial F}{\partial y_{j}} & =-\frac{d_{j} y_{j}^{\alpha-1}}{[a(\boldsymbol{y})]^{2}}, \quad 1 \leq j \leq s, \\
\frac{\partial^{2} F}{\partial y_{j} y_{k}} & =\frac{2 d_{j} d_{k}\left(y_{j} y_{k}\right)^{\alpha-1}}{[a(\boldsymbol{y})]^{3}}, \quad 1 \leq j, k \leq s, j \neq k,
\end{aligned}
$$

and, for the mixed first partial derivative with respect to the variables with labels in $\mathfrak{u}$,

$$
\frac{\partial^{|\mathfrak{u}|} F}{\partial \boldsymbol{y}_{\mathfrak{u}}}=\frac{|\mathfrak{u}| ! \prod_{j \in \mathfrak{u}}\left(-d_{j} y_{j}^{\alpha-1}\right)}{[a(\boldsymbol{y})]^{|\mathfrak{u}|+1}} .
$$

Hence, on using $a(\boldsymbol{y}) \geq 1$ we find, for $\alpha>1 / 2$,

$$
\int_{[0,1]^{\mathfrak{u} \mid}}\left|\frac{\partial^{|\mathfrak{u}|} F}{\partial \boldsymbol{y}_{\mathfrak{u}}}\left(\boldsymbol{y}_{\mathfrak{u}}, 1\right)\right|^{2} d \boldsymbol{y}_{\mathfrak{u}} \leq \frac{(|\mathfrak{u}| !)^{2} \prod_{j \in \mathfrak{u}} d_{j}^{2}}{(2 \alpha-1)^{|\mathfrak{u}|}},
$$


and on defining $b_{j}:=d_{j} /(\sqrt{2 \alpha-1})=\alpha /\left(j^{2} \sqrt{2 \alpha-1}\right)$ we obtain a bound on the norm (1.7),

$$
\|F\|_{s, \gamma}^{2} \leq \sum_{\mathfrak{u} \subseteq\{1: s\}} \frac{(|\mathfrak{u}| !)^{2} \prod_{j \in \mathfrak{u}} b_{j}^{2}}{\gamma_{\mathfrak{u}}}
$$

We observe that as $\alpha \rightarrow 1 / 2$, the bound approaches infinity, even though the expectation of $1 / a(y)$ is well defined for all $0<\alpha \leq 1$. We thus see that non-Hilbert space norms might be advantageous in order to cover as wide a class of integrands as possible. This is one motivation for considering the Banach space setting in Section 3.

Given the bound (1.18) on the norm, how can we decide on the best choice of weights $\gamma_{\mathfrak{u}}$ and go on to obtain an error bound for $\left|I_{s}(F)-Q_{s, N}(F)\right|$ ? The principle used in our other paper [31] is that we should choose weights that as far as possible minimize the error bound (1.5). Bearing in mind the upper bound (1.18), this means that we should choose weights that minimize the right-hand side of

$$
\left|I_{s}(F)-Q_{s, N}(F)\right| \leq e^{\operatorname{wor}}\left(\mathcal{P} ; \mathcal{H}_{s, \gamma}\right)\left(\sum_{\mathfrak{u} \subseteq\{1: s\}} \frac{(|\mathfrak{u}| !)^{2} \prod_{j \in \mathfrak{u}} b_{j}^{2}}{\gamma_{\mathfrak{u}}}\right)^{1 / 2} .
$$

We show in Section 4 (see Theorem 4.1) that if $Q_{s, N}$ is a randomly shifted lattice rule constructed by the CBC algorithm, and if we interpret the error in (1.19) as the root-mean-square error averaged over shifts, then the right-hand side of (1.19) can be further bounded (for the simplest case of prime $N$ ) by

$$
\left(\frac{2}{N} \sum_{\emptyset \neq \mathfrak{u} \subseteq\{1: s\}} \gamma_{\mathfrak{u}}^{\lambda}(\rho(\lambda))^{|\mathfrak{u}|}\right)^{1 /(2 \lambda)}\left(\sum_{\mathfrak{u} \subseteq\{1: s\}} \frac{(|\mathfrak{u}| !)^{2} \prod_{j \in \mathfrak{u}} b_{j}^{2}}{\gamma_{\mathfrak{u}}}\right)^{1 / 2},
$$

where

$$
\rho(\lambda):=\frac{2 \zeta(2 \lambda)}{\left(2 \pi^{2}\right)^{\lambda}}+\left(\frac{1}{3}\right)^{\lambda} .
$$

Here $\zeta(x):=\sum_{k=1}^{\infty} 1 / k^{x}$, with $x>1$, is the Riemann zeta function, and $\lambda$ is any number satisfying $1 / 2<\lambda \leq 1$. Obviously, we get the best rate of convergence, a rate close to $1 / N$, by taking $\lambda$ close to $1 / 2$; but $\lambda$ must remain strictly greater than $1 / 2$ because $\zeta(x) \rightarrow \infty$ as $x \rightarrow 1$.

In our other paper [31] it is shown (and the reader can verify by simple calculus) that (1.20) is minimized, and bounded independently of $s$, by choosing the weights to be

$$
\gamma_{\mathfrak{u}}=\left(|\mathfrak{u}| ! \prod_{j \in \mathfrak{u}} \frac{b_{j}}{\sqrt{\rho(\lambda)}}\right)^{2 /(1+\lambda)} .
$$

Thus the best choice of weights, in the sense of minimizing the error bound, is a POD (product and order dependent) weight, and this choice gives a rate of convergence that is independent of $s$ and arbitrarily close to $1 / N$ when $\lambda$ is close to $1 / 2$. 


\section{QMC in a Hilbert space setting}

In the standard setting, as described in Section 1.1, the function space $\mathcal{H}_{s, \gamma}$ is a special kind of Hilbert space called a reproducing kernel Hilbert space, or RKHS [1]. Such Hilbert spaces are often useful in numerical analysis, since a Hilbert space is an RKHS if and only if point evaluation is a bounded linear functional. It turns out that the error analysis for numerical integration in general, and QMC integration in particular, is especially simple in an RKHS setting. In this section we describe that error analysis and then apply it to the standard setting.

2.1. Reproducing kernel Hilbert spaces A Hilbert space $\mathcal{H}$ with inner product $\langle\cdot, \cdot\rangle_{\mathcal{H}}$ is an RKHS [1] if and only if there is a unique function $K:[0,1]^{s} \times[0,1]^{s} \rightarrow \mathbb{R}$, referred to as the reproducing kernel, with the following properties:

$$
\begin{aligned}
& K(\boldsymbol{y}, \cdot) \in \mathcal{H} \quad \text { for all } \boldsymbol{y} \in[0,1]^{s}, \\
& K\left(\boldsymbol{y}, \boldsymbol{y}^{\prime}\right)=K\left(\boldsymbol{y}^{\prime}, \boldsymbol{y}\right) \quad \text { for all } \boldsymbol{y}, \boldsymbol{y}^{\prime} \in[0,1]^{s}, \\
& F(\boldsymbol{y})=\langle F, K(\boldsymbol{y}, \cdot)\rangle_{\mathcal{H}} \quad \text { for all } \boldsymbol{y} \in[0,1]^{s}, F \in \mathcal{H} .
\end{aligned}
$$

The last property is known as the reproducing property. The existence of the reproducing kernel is a consequence of the boundedness of point evaluation, as follows from the Riesz representation theorem [1].

The RKHS approach provides a very powerful tool for obtaining QMC error bounds, provided that the kernel is known and available in closed form (which is certainly the case for the standard setting; see equation (2.4) below). This is because, as we now show, there is a simple but completely general expression for the worst-case error in terms of the reproducing kernel. Thus the worst-case error in an RKHS has an explicit formula.

2.2. Worst-case error in an RKHS Suppose that $\mathcal{H}$ is an RKHS with reproducing kernel $K:[0,1]^{s} \times[0,1]^{s} \rightarrow \mathbb{R}$. Using (1.2) and the reproducing property (2.1), we can write

$$
Q_{s, N}(F)=\frac{1}{N} \sum_{i=1}^{N}\left\langle F, K\left(\boldsymbol{y}^{(i)}, \cdot\right)\right\rangle_{\mathcal{H}}=\left\langle F, \frac{1}{N} \sum_{i=1}^{N} K\left(\boldsymbol{y}^{(i)}, \cdot\right)\right\rangle_{\mathcal{H}} .
$$

In a similar way, any bounded linear functional $T$ on $\mathcal{H}$ can be represented as $T(F)=$ $\langle F, \tilde{T}\rangle_{\mathcal{H}}$, with $\tilde{T}(\boldsymbol{y})=T(K(\boldsymbol{y}, \cdot))$ for all $\boldsymbol{y} \in[0,1]^{s}$. Assuming that $I_{s}$ is a bounded linear functional on $\mathcal{H}$ (which will always be the case for the spaces of interest to us), we can thus write

$$
I_{s}(F)=\left\langle F, \int_{[0,1]^{s}} K(\boldsymbol{y}, \cdot) d \boldsymbol{y}\right\rangle_{\mathcal{H}} .
$$

Hence, by subtraction,

$$
I_{s}(F)-Q_{s, N}(F)=\left\langle F, \xi_{s, N}\right\rangle_{H},
$$


where $\xi_{s, N}$ is the representer of the error,

$$
\begin{aligned}
\xi_{s, N}\left(\boldsymbol{y}^{\prime}\right) & :=I_{s}\left(K\left(\cdot, \boldsymbol{y}^{\prime}\right)\right)-Q_{s, N}\left(K\left(\cdot, \boldsymbol{y}^{\prime}\right)\right) \\
& =\int_{[0,1]^{s}} K\left(\boldsymbol{y}, \boldsymbol{y}^{\prime}\right) d \boldsymbol{y}-\frac{1}{N} \sum_{i=1}^{N} K\left(\boldsymbol{y}^{(i)}, \boldsymbol{y}^{\prime}\right) .
\end{aligned}
$$

By the Cauchy-Schwarz inequality, we obtain from (2.2) the error bound

$$
\left|I_{s}(F)-Q_{s, N}(F)\right|=\left|\left\langle F, \xi_{s, N}\right\rangle_{\mathcal{H}}\right| \leq\|F\|_{\mathcal{H}}\left\|\xi_{s, N}\right\|_{\mathcal{H}} .
$$

Equality holds when $F$ is a multiple of $\xi_{s, N}$, and thus, by the definition (1.4) of worstcase error,

$$
e^{\text {wor }}(\mathcal{P} ; \mathcal{H})=\left\|\xi_{s, N}\right\|_{\mathcal{H}}
$$

This leads us to the following explicit formula for the worst-case error.

LEMma 2.1. If $\mathcal{H}$ is an RKHS with reproducing kernel $K$ and $I_{s}$ is a bounded linear functional on $\mathcal{H}$, then

$$
\begin{aligned}
{\left[e^{\text {wor }}(\mathcal{P} ; \mathcal{H})\right]^{2}=} & \int_{[0,1]^{s}}\left(\int_{[0,1]^{s}} K\left(\boldsymbol{y}, \boldsymbol{y}^{\prime}\right) d \boldsymbol{y}\right) d \boldsymbol{y}^{\prime} \\
& -\frac{2}{N} \sum_{i=1}^{N} \int_{[0,1]^{s}} K\left(\boldsymbol{y}^{(i)}, \boldsymbol{y}\right) d \boldsymbol{y}+\frac{1}{N^{2}} \sum_{i=1}^{N} \sum_{i^{\prime}=1}^{N} K\left(\boldsymbol{y}^{(i)}, \boldsymbol{y}^{\left(i^{\prime}\right)}\right) .
\end{aligned}
$$

Proof. Using the reproducing property of $K(\cdot, \cdot)$ and the linearity of $I_{S}(\cdot)$ and $Q_{s, N}(\cdot)$,

$$
\begin{aligned}
& {\left[e^{\text {wor }}(\mathcal{P} ; \mathcal{H})\right]^{2} } \\
&=\left\|\int_{[0,1]^{s}} K(\boldsymbol{y}, \cdot) d \boldsymbol{y}-\frac{1}{N} \sum_{i=1}^{N} K\left(\boldsymbol{y}^{(i)}, \cdot\right)\right\|_{\mathcal{H}}^{2} \\
&=\left\langle\int_{[0,1]^{s}} K(\boldsymbol{y}, \cdot) d \boldsymbol{y}-\frac{1}{N} \sum_{i=1}^{N} K\left(\boldsymbol{y}^{(i)}, \cdot\right), \int_{[0,1]^{s}} K(\boldsymbol{y}, \cdot) d \boldsymbol{y}-\frac{1}{N} \sum_{i=1}^{N} K\left(\boldsymbol{y}^{(i)}, \cdot\right)\right\rangle_{\mathcal{H}} \\
&=\left\langle\int_{[0,1]^{s}} K(\boldsymbol{y}, \cdot) d \boldsymbol{y}, \int_{[0,1]^{s}} K(\boldsymbol{y}, \cdot) d \boldsymbol{y}\right\rangle_{\mathcal{H}}-\frac{2}{N} \sum_{i=1}^{N}\left\langle\int_{[0,1]^{s}} K(\boldsymbol{y}, \cdot) d \boldsymbol{y}, K\left(\boldsymbol{y}^{(i)}, \cdot\right)\right\rangle_{\mathcal{H}} \\
&+\frac{1}{N^{2}} \sum_{i=1}^{N} \sum_{i^{\prime}=1}^{N}\left\langle K\left(\boldsymbol{y}^{(i)}, \cdot\right), K\left(\boldsymbol{y}^{\left(i^{\prime}\right)}, \cdot\right)\right\rangle_{\mathcal{H}}
\end{aligned}
$$

Using again the reproducing property (2.1), we obtain the desired formula.

2.3. Error analysis in the standard setting Recall that $\mathcal{H}_{s, \gamma}$ denotes the Hilbert space in the standard setting of Section 1.1. Then the inner product corresponding to the norm (1.7) is

$$
\langle F, G\rangle_{s, \gamma}=\sum_{\mathfrak{u} \subseteq\{1: s\}} \gamma_{\mathfrak{u}}^{-1} \int_{[0,1]^{|\mathfrak{u}|}} \frac{\partial^{|\mathfrak{u}|} F}{\partial \boldsymbol{y}_{\mathfrak{u}}}\left(\boldsymbol{y}_{\mathfrak{u}}, 1\right) \frac{\partial^{|\mathfrak{u}|} G}{\partial \boldsymbol{y}_{\mathfrak{u}}}\left(\boldsymbol{y}_{\mathfrak{u}}, 1\right) d \boldsymbol{y}_{\mathfrak{u}},
$$

where $\gamma_{\mathfrak{u}}$ is again given by (1.8) and the notation is as in (1.7). 
It can be shown that the reproducing kernel for the space $\mathcal{H}_{s, \gamma}$ is

$$
K_{s, \gamma}\left(\boldsymbol{y}, \boldsymbol{y}^{\prime}\right)=\prod_{j=1}^{s}\left(1+\gamma_{j}\left[1-\max \left(y_{j}, y_{j}^{\prime}\right)\right]\right), \quad \boldsymbol{y}, \boldsymbol{y}^{\prime} \in[0,1]^{s} .
$$

(It is a useful exercise, based solely on integration by parts and the definition of the norm, to verify the reproducing property (2.1) for $s=1$.) An explicit expression for the worst-case error of the space $\mathcal{H}_{s, \gamma}$ is then given by Lemma 2.1 as

$$
\begin{array}{r}
{\left[e^{\text {wor }}\left(\mathcal{P} ; \mathcal{H}_{s, \gamma}\right)\right]^{2}=\prod_{j=1}^{s}\left(1+\frac{\gamma_{j}}{3}\right)-\frac{2}{N} \sum_{i=1}^{N} \prod_{j=1}^{s}\left(1+\frac{\gamma_{j}}{2}\left(1-\left[t_{j}^{(i)}\right]^{2}\right)\right)} \\
+\frac{1}{N^{2}} \sum_{i=1}^{N} \sum_{i^{\prime}=1}^{N} \prod_{j=1}^{s}\left(1+\gamma_{j}\left(1-\max \left(t_{j}^{(i)}, t_{j}^{\left(i^{\prime}\right)}\right)\right)\right) .
\end{array}
$$

As we explain in Section 3, this quantity is also known by the name "weighted $L^{2}$ discrepancy". (The unweighted version, where $\gamma_{j}=1$, is due to Warnock [60]; the weighted version was derived by Joe [25].)

This machinery provided the foundation for the series of developments foreshadowed in Sections 1.1 and 1.2, from the nonconstructive error bounds on general QMC methods to the component-by-component (CBC) construction of randomly shifted lattice rules that achieve the $c_{\delta} / N^{1-\delta}$ bound with $\delta>0$ arbitrarily small (probabilistically, since the shift is random) under the condition (1.10). More details about the theory and construction of lattice rules are given in Sections 4 and 5 .

\section{Banach space settings with general weights}

In this section we go beyond the standard setting in two ways. Firstly, we move away from Hilbert space settings to Banach space settings. Secondly, we consider general weights instead of the product weights (1.8).

More precisely, we assume that the integrand $F$ in (1.1) admits, in particular, mixed first derivatives belonging to $L^{q}$ for some $q \in[1, \infty]$, and we define a norm by combining the derivative terms in the $\ell^{r}$ sense for $r \in[1, \infty]$. Denoting here the function space by $\mathcal{W}_{s, \gamma}^{q, r}$, we derive the weighted Koksma-Hlawka inequality

$$
\left|I_{s}(F)-Q_{s, N}(F)\right| \leq D_{s, \gamma}^{q^{\prime}, r^{\prime}}(\mathcal{P})\|F\|_{\mathcal{W}_{s, \gamma}^{q, r},}
$$

where $q^{\prime}$ and $r^{\prime}$ are the Hölder conjugates of $q$ and $r$, respectively; that is, $1 / q+1 / q^{\prime}=1$ and $1 / r+1 / r^{\prime}=1$ for $q$ and $r$ strictly between 1 and $\infty$, and as usual 1 is the conjugate of $\infty$ and vice versa. For the precise definitions of the norm $\|F\|_{\mathcal{W}_{s, \gamma}^{q, r}}$ and the discrepancy $D_{s, \gamma}^{q^{\prime}, r^{\prime}}(\mathcal{P})$, see equations (3.4) and (3.5) below. For now, it suffices to say that the case where $q=r=2$ corresponds to the standard setting if we have product weights, while the case where $q=r=1$ corresponds to the classical setting if all weights are equal to 1 .

We explain below that $q$ and $r$ play different roles in the QMC error bounds, and that decoupling $q$ and $r$ allows for more flexibility in the QMC analysis. 
3.1. Deriving the weighted Koksma-Hlawka inequality In this subsection we derive the generalized QMC error bound (3.1), a weighted version of the KoksmaHlawka inequality (1.12). Like the Koksma-Hlawka inequality itself, this can be derived from the Hlawka-Zaremba identity [24, 61]. Assuming that the integrand $F$ in (1.1) is sufficiently smooth, the identity states that

$$
I_{S}(F)-Q_{s, N}(F)=\sum_{\emptyset \neq \mathfrak{u} \subseteq\{1: s\}}(-1)^{|\mathfrak{u}|} \int_{[0,1]^{|\mathfrak{u}|}} \frac{\partial^{|\mathfrak{u}|} F}{\partial \boldsymbol{y}_{\mathfrak{u}}}\left(\boldsymbol{y}_{\mathfrak{u}}, 1\right) \operatorname{discr} \mathcal{P}\left(\boldsymbol{y}_{\mathfrak{u}}, 1\right) d \boldsymbol{y},
$$

where $\operatorname{discr}_{\mathcal{P}}(\cdot)$ is the local discrepancy function defined in (1.13) and the notation is the same as in (1.7).

The validity of the Hlawka-Zaremba identity is easily verified in the $s=1$ case: if we adopt the convention that

$$
0=y^{(0)} \leq y^{(1)} \leq \cdots \leq y^{(N)} \leq y^{(N+1)}=1,
$$

then the right-hand side of the identity becomes

$$
\begin{aligned}
-\int_{0}^{1} F^{\prime}(y)\left(y-\frac{\left|i: y^{(i)} \in[0, y)\right|}{N}\right) d y & =-\sum_{i=1}^{N+1} \int_{y^{(i-1)}}^{y^{(i)}} F^{\prime}(y)\left(y-\frac{i-1}{N}\right) d y \\
& =-\frac{1}{N} \sum_{i=1}^{N} F\left(y^{(i)}\right)+\int_{0}^{1} F(y) d y,
\end{aligned}
$$

where the last step comes from integration by parts and recombination of the terms. The general case follows in a similar way by recursively applying the preceding univariate integration by parts with respect to each coordinate $y_{j}$.

As in (2.3), we introduce weights $\gamma_{\mathfrak{u}}$ into the error bound, but now allowing a different positive weight $\gamma_{\mathfrak{u}}$ for each subset $\mathfrak{u} \subseteq\{1: s\}$. For each term of the sum in (3.2), we multiply and divide by $\gamma_{\mathfrak{u}}^{1 / 2}$ to obtain

$$
I_{s}(F)-Q_{s, N}(F)=\sum_{\emptyset \neq \mathfrak{u} \subseteq\{1: s\}}(-1)^{|\mathfrak{u}|} \int_{[0,1]^{|\mathfrak{u}|}} \frac{\partial^{|\mathfrak{u}|} F}{\partial \boldsymbol{y}_{\mathfrak{u}}}\left(\boldsymbol{y}_{\mathfrak{u}}, 1\right) \gamma_{\mathfrak{u}}^{-1 / 2} \gamma_{\mathfrak{u}}^{1 / 2} \operatorname{discr} \mathcal{P}\left(\boldsymbol{y}_{\mathfrak{u}}, 1\right) d \boldsymbol{y}_{\mathfrak{u}} .
$$

Clearly, nothing has been changed at this point. Next, we use Hölder's inequality for integrals with the conjugate exponents $q$ and $q^{\prime}$ to obtain

$$
\left|I_{S}(F)-Q_{s, N}(F)\right| \leq \sum_{\emptyset \neq \mathfrak{u} \subseteq\{1: s\}}\left\|\gamma_{\mathfrak{u}}^{-1 / 2} \frac{\partial^{|\mathfrak{u}|} F}{\partial \boldsymbol{y}_{\mathfrak{u}}}\left(\boldsymbol{y}_{\mathfrak{u}}, 1\right)\right\|_{L^{q}}\left\|\gamma_{\mathfrak{u}}^{1 / 2} \operatorname{discr} \mathcal{P}\left(\boldsymbol{y}_{\mathfrak{u}}, 1\right)\right\|_{L^{q^{\prime}}},
$$

where the $L^{q}$ norm of a function $f$ is defined as usual by

$$
\|f\|_{L^{q}}:= \begin{cases}\left(\int_{[0,1]^{s}}|f(\boldsymbol{y})|^{q} d \boldsymbol{y}\right)^{1 / q} & \text { if } 1 \leq q<\infty \\ \operatorname{ess~sup~}_{\boldsymbol{y} \in[0,1]^{s}}|f(\boldsymbol{y})| & \text { if } q=\infty .\end{cases}
$$


Then we use Hölder's inequality for sums with the conjugate pair $r$ and $r^{\prime}$ to arrive at

$$
\begin{aligned}
\left|I_{s}(F)-Q_{s, N}(F)\right| \leq & \left(\sum_{\emptyset \neq \mathfrak{u} \subseteq\{1: s\}}\left\|\gamma_{\mathfrak{u}}^{-1 / 2} \frac{\partial^{|\mathfrak{u}|} F}{\partial \boldsymbol{y}_{\mathfrak{u}}}\left(\boldsymbol{y}_{\mathfrak{u}}, 1\right)\right\|_{L^{q}}^{r}\right)^{1 / r} \\
& \times\left(\sum_{\emptyset \neq \mathfrak{u} \subseteq\{1: s\}}\left\|\gamma_{\mathfrak{u}}^{1 / 2} \operatorname{discr} \mathcal{P}\left(\boldsymbol{y}_{\mathfrak{u}}, 1\right)\right\|_{L^{q^{\prime}}}^{r^{\prime}}\right)^{1 / r^{\prime}},
\end{aligned}
$$

with the usual modification for the case where $r=\infty$ or $r^{\prime}=\infty$.

The first factor in (3.3) prompts us to define our norm, for $1 \leq q \leq \infty$, by

$$
\|F\|_{\mathcal{W}_{s, \gamma}^{q, r}}:= \begin{cases}\left(\sum_{\mathfrak{u} \subseteq\{1: s\}}\left\|\gamma_{\mathfrak{u}}^{-1 / 2} \frac{\partial^{|\mathfrak{u}|} F}{\partial \boldsymbol{y}_{\mathfrak{u}}}\left(\boldsymbol{y}_{\mathfrak{u}}, 1\right)\right\|_{L^{q}}^{r}\right)^{1 / r} & \text { if } 1 \leq r<\infty \\ \max _{\mathfrak{u} \subseteq\{1: s\}}\left\|\gamma_{\mathfrak{u}}^{-1 / 2} \frac{\partial^{|\mathfrak{u}|} F}{\partial \boldsymbol{y}_{\mathfrak{u}}}\left(\boldsymbol{y}_{\mathfrak{u}}, 1\right)\right\|_{L^{q}} & \text { if } r=\infty,\end{cases}
$$

and we denote by $\mathcal{W}_{s, \gamma}^{q, r}$ the completion of the space $C^{\infty}\left([0,1]^{s}\right)$ under this norm. Note that we have added the $\mathfrak{u}=\emptyset$ term to the sum to make it a true norm. The expression without the $\mathfrak{u}=\emptyset$ term is a seminorm which is sometimes referred to as the variation of $F$. We define the second factor in (3.3) to be the weighted discrepancy of the QMC point set $\mathcal{P}$,

$$
D_{s, \gamma}^{q^{\prime}, r^{\prime}}(\mathcal{P}):= \begin{cases}\left(\sum_{\emptyset \neq \mathfrak{u} \subseteq\{1: s\}}\left\|\gamma_{\mathfrak{u}}^{1 / 2} \operatorname{discr} \mathcal{P}\left(\boldsymbol{y}_{\mathfrak{u}}, 1\right)\right\|_{L^{q^{\prime}}}^{r^{\prime}}\right)^{1 / r^{\prime}} & \text { if } 1 \leq r^{\prime}<\infty \\ \max _{\emptyset \neq \mathfrak{u} \subseteq\{1: s\}}\left\|\gamma_{\mathfrak{u}}^{1 / 2} \operatorname{discr} \mathcal{P}\left(\boldsymbol{y}_{\mathfrak{u}}, 1\right)\right\|_{L^{q^{\prime}}} & \text { if } r^{\prime}=\infty .\end{cases}
$$

With the definitions (3.4) and (3.5), we obtain (3.1).

The weighted Koksma-Hlawka inequality (3.1) was first derived by Sloan and Woźniakowski [53], but with $q$ always equal to $r$. The unweighted version, namely the one with all weights $\gamma_{\mathfrak{u}}=1$, had appeared earlier in the QMC literature, but again seemingly always with $q=r$; it was derived by Zaremba [61] for $q=r=2$ and by Sobol' [56] for general $q=r$. The classical Koksma-Hlawka inequality can be recovered by taking the unweighted version with $q=r=1$; it was proved by Koksma [28] for dimension $s=1$ and generalized by Hlawka [23] for $s \geq 1$. Actually, the classical Koksma-Hlawka inequality in its original form has the variation in the sense of Hardy and Krause instead of the norm of $F$; this variation is precisely the norm without the $\mathfrak{u}=\emptyset$ term whenever all mixed first partial derivatives are continuous on $[0,1]^{s}$. For more details see, for example, the book by Niederreiter [36].

As an exercise, the reader may wish to check for which parameters $q$ and $r$ and which weights $\gamma_{\mathfrak{u}}$ the function $F(y)$ in Section 1.5 belongs to $\mathcal{W}_{s, \gamma}^{q, r}$ and has its norm bounded independently of $s$. 
3.2. Connection between discrepancy and worst-case error It turns out that the weighted discrepancy $D_{s, \gamma}^{q^{\prime}, r^{\prime}}(\mathcal{P})$ is precisely the worst-case error in the space $\mathcal{W}_{s, \gamma}^{q, r}$, indicating that the weighted Koksma-Hlawka inequality is tight.

Lemma 3.1. Let $\mathcal{P}$ be a finite point set in $[0,1]^{s}$. Then

$$
e^{\mathrm{wor}}\left(\mathcal{P} ; \mathcal{W}_{s, \gamma}^{q, r}\right)=D_{s, \gamma}^{q^{\prime}, r^{\prime}}(\mathcal{P})
$$

Proof. For a function $F$ in the unit ball of $\mathcal{W}_{s, \gamma}^{q, r}$, the weighted Koksma-Hlawka inequality (3.1) yields

$$
\left|I_{s}(F)-Q_{s, N}(F)\right| \leq D_{s, \gamma}^{q^{\prime}, r^{\prime}}(\mathcal{P}),
$$

so to prove the lemma it would be sufficient to construct an integrand for which equality is attained in (3.6). This is possible for $q, r \geq 2$. For $q=1$ or $r=1$ (that is, $q^{\prime}=\infty$ or $r^{\prime}=\infty$ ), instead a "nearly worst-case" integrand $F^{\epsilon}$ is constructed for arbitrary $\epsilon>0$, one for which

$$
\left|I_{s}\left(F^{\epsilon}\right)-Q_{s, N}\left(F^{\epsilon}\right)\right| \geq D_{s, \gamma}^{q^{\prime}, r^{\prime}}(\mathcal{P})-\epsilon .
$$

Under more general function space settings, this result is discussed by Hickernell [13] for $q=r$, and for general $q$ and $r$ by Hickernell et al. [17], who also give proofs for $q=r=1$ [16] and $q, r \geq 2$ [19].

3.3. Two special cases: $q=r=2$ and $q=r=1$ Most QMC analyses follow one of two approaches. The first approach uses the Hilbert space setting $q=r=2$ and studies the weighted $L^{2}$ discrepancy $D_{s, \gamma}^{2,2}(\mathcal{P})$. This has the nice explicit representation

$$
\begin{aligned}
{\left[D_{s, \gamma}^{2,2}(\mathcal{P})\right]^{2}=\sum_{\emptyset \neq \mathfrak{u} \subseteq\{1: s\}} \gamma_{\mathfrak{u}}\left[\frac{1}{3^{|\mathfrak{u}|}}\right.} & -\frac{2}{N} \sum_{i=1}^{N} \prod_{j \in \mathfrak{u}} \frac{1-\left[t_{j}^{(i)}\right]^{2}}{2} \\
& \left.+\frac{1}{N^{2}} \sum_{i=1}^{N} \sum_{i^{\prime}=1}^{N} \prod_{j \in \mathfrak{u}}\left(1-\max \left(t_{j}^{(i)}, t_{j}^{\left(i^{\prime}\right)}\right)\right)\right],
\end{aligned}
$$

which we already met for the product weight case as a worst-case error in (2.5). Two QMC constructions related to this formula are given by Sloan et al. [49, 50].

The other important approach uses the non-Hilbert setting of $q=r=1$ (and thus $\left.q^{\prime}=r^{\prime}=\infty\right)$ and so studies the weighted star discrepancy $[6,22,26,53,59]$

$$
\begin{aligned}
D_{s, \gamma}^{\infty, \infty}(\mathcal{P}) & =\max _{\emptyset \neq \mathfrak{u} \subseteq\{1: s\}} \sup _{\boldsymbol{y}_{\mathfrak{u}} \in[0,1]^{|\mathfrak{l}|}} \gamma_{\mathfrak{u}}^{1 / 2} \operatorname{discr} \mathcal{P}\left(\boldsymbol{y}_{\mathfrak{u}}, 1\right) \\
& =\sup _{\boldsymbol{y} \in[0,1]^{s}} \max _{\emptyset \neq \mathfrak{u} \subseteq\{1: s\}} \gamma_{\mathfrak{u}}^{1 / 2} \operatorname{discr} \mathcal{P}\left(\boldsymbol{y}_{\mathfrak{u}}, 1\right)=: D_{s, \gamma}^{*}(\mathcal{P}) .
\end{aligned}
$$

(We remark that our scaling with weights $\gamma_{\mathfrak{u}}^{1 / 2}$ is consistent with that of Sloan and Woźniakowski [53] and Wang [59], but the scaling $\gamma_{\mathfrak{u}}$ was used by Dick and Pillichshammer [6] and Joe [26].) Unlike the $L^{2}$ counterpart, there is no easy formula 
for computing the weighted star discrepancy for a given point set (except when the dimensionality $s$ is as low as 2 or 3 ), and one must work with some form of upper bound. We discuss recent constructive results from both approaches in Section 4.

3.4. The benefit of decoupling $q$ and $\boldsymbol{r}$ The idea of decoupling $q$ and $r$ originated from the works of Hickernell et al. [17, 19], who observed that since the $L^{q}$ norm for a function defined on the unit cube increases with increasing $q$ and the $\ell^{r}$ norm for a vector increases with decreasing $r$, we have the partial ordering

$$
\begin{gathered}
\|F\|_{\mathcal{W}_{s, \gamma}^{1, \infty}} \leq\|F\|_{\mathcal{W}_{s, \gamma}^{q_{1}, r_{1}}} \leq\|F\|_{\mathcal{W}_{s, \gamma}^{q_{2}, r_{2}}} \leq\|F\|_{\mathcal{W}_{s, \gamma}^{\infty, 1}}, \\
D_{s, \gamma}^{\infty, 1}(\mathcal{P}) \geq D_{s, \gamma}^{q_{1}^{\prime}, r_{1}^{\prime}}(\mathcal{P}) \geq D_{s, \gamma}^{q_{2}^{\prime}, r_{2}^{\prime}}(\mathcal{P}) \geq D_{s, \gamma}^{1, \infty}(\mathcal{P})
\end{gathered}
$$

for all

$$
\left\{\begin{array} { l } 
{ 1 \leq q _ { 1 } \leq q _ { 2 } \leq \infty , } \\
{ \infty \geq r _ { 1 } \geq r _ { 2 } \geq 1 , }
\end{array} \quad \left\{\begin{array}{l}
\infty \geq q_{1}^{\prime} \geq q_{2}^{\prime} \geq 1, \\
1 \leq r_{1}^{\prime} \leq r_{2}^{\prime} \leq \infty
\end{array}\right.\right.
$$

This partial ordering implies the embedding

$$
\mathcal{W}_{s, \gamma}^{\infty, 1} \subset \mathcal{W}_{s, \gamma}^{q_{2}, r_{2}} \subset \mathcal{W}_{s, \gamma}^{q_{1}, r_{1}} \subset \mathcal{W}_{s, \gamma}^{1, \infty}
$$

with continuous injections.

Thus, in the weighted Koksma-Hlawka inequality (3.1), we are able to have a smaller norm paired with a larger discrepancy, or a larger norm paired with a smaller discrepancy. The trade-off between the norm and the discrepancy determines the final QMC error bound. It is therefore important to consider the norm and the discrepancy together, rather than focusing solely on the discrepancy (as is done in many QMC analyses).

We also see that decoupling $q$ and $r$ allows for more flexibility in the QMC analysis. Observe that the partial ordering (3.8) does not allow for a comparison between $D_{s, \gamma}^{2,2}(\mathcal{P})$ and $D_{s, \gamma}^{\infty, \infty}(\mathcal{P})$ in general; thus the two special cases discussed in Section 3.3 have been treated separately in the past. However, if a given integrand can be bounded in a larger norm, say $\mathcal{W}_{s, \gamma}^{\infty, 1}$, then the smaller discrepancy $D_{s, \gamma}^{1, \infty}(\mathcal{P})$ can be used, and in this case all existing bounds on other larger discrepancies, including both $D_{s, \gamma}^{2,2}(\mathcal{P})$ and $D_{s, \gamma}^{\infty, \infty}(\mathcal{P})$, can be applied.

3.5. The weighted space $\boldsymbol{W}_{s, \gamma}^{q, r}$ and generalizations It is easy to see that different values of the parameter $r$ yield equivalent norms in the function space $\mathcal{W}_{s, \gamma}^{q, r}$ and thus do not change the function space itself. However, as we see in Section 3.9, tractability results can depend on the specific value of $r$.

In the norm (3.4), $\partial^{|\mathfrak{u}|} F / \partial \boldsymbol{y}_{\mathfrak{u}}$ is evaluated at $\left(\boldsymbol{y}_{\mathfrak{u}}, 1\right)$, meaning that the components of $\boldsymbol{y}$ with indices outside the set $\mathfrak{u}$ are replaced by 1 . We could instead use an arbitrary anchor $a \in[0,1]$ (the choice $a=1 / 2$ is popular). There is also an unanchored variant which, instead of freezing a component at the anchor value, integrates a component out [52]. For simplicity, we consider in this paper only the anchored space and only the anchor $a=1$. 
Since the integrals arising from practical problems are often formulated over $\mathbb{R}^{s}$ rather than over the unit cube, Kuo et al. [32] considered a generalization of the function space for unbounded integrands in $\mathbb{R}^{s}$. Earlier works by Hickernell $[13,14]$ considered unweighted spaces with general $q=r$, with a generalization of the function space that covers both the anchored and unanchored variants. Later works by Hickernell et al. [16-19] considered weighted spaces with general anchor, general product domain, and general product measure, for the case $q=r=1[16,18]$ and also for general $q$ and $r[17,19]$.

3.6. Anchored decomposition Let $\mathcal{W}_{\mathfrak{u}, \gamma}^{q, r}$ denote the subspace of $\mathcal{W}_{s, \gamma}^{q, r}$ consisting of functions $F(\boldsymbol{y})$ that depend only on the set of variables whose indices belong to a set $\mathfrak{u} \subseteq\{1: s\}$. Denote this set of variables by $\boldsymbol{y}_{\mathfrak{u}}$. Every function in $\mathcal{W}_{s, \gamma}^{q, r}$ has a unique decomposition of the form

$$
F(y)=\sum_{\mathfrak{u} \subseteq\{1: s\}} F_{\mathfrak{u}}\left(y_{\mathfrak{u}}\right),
$$

where $F_{\mathfrak{u}}$ belongs to the space $\mathcal{W}_{\mathfrak{u}, \gamma}^{q, r}$ and satisfies also the condition that for all $\mathfrak{u} \neq \emptyset$,

$$
F_{\mathfrak{u}}\left(\boldsymbol{y}_{\mathfrak{u}}\right)=0 \quad \text { if } \quad y_{j}=1 \text { for any } j \in \mathfrak{u} .
$$

This is sometimes called the anchored decomposition. It was shown by Kuo et al. [33] that an explicit formula for $F_{\mathfrak{u}}$ is given by

$$
F_{\mathfrak{u}}\left(\boldsymbol{y}_{\mathfrak{u}}\right)=\sum_{\mathfrak{v} \subseteq \mathfrak{u}}(-1)^{|\mathfrak{u}|-|\mathfrak{p}|} F\left(\boldsymbol{y}_{\mathfrak{v}}, 1\right) .
$$

Similar decompositions exist for a general anchor and other more general variants of the function space [33]. The best known of these decompositions is the ANOVA decomposition [3, 57].

From the anchored decomposition of $F$ we find that

$$
\frac{\partial^{|\mathfrak{u}|} F}{\partial \boldsymbol{y}_{\mathfrak{u}}}\left(\boldsymbol{y}_{\mathfrak{u}}, 1\right)=\sum_{\mathfrak{u}^{\prime} \subseteq\{1: s\}} \frac{\partial^{|\mathfrak{u}|} F_{\mathfrak{u}^{\prime}}}{\partial \boldsymbol{y}_{\mathfrak{u}}}\left(\boldsymbol{y}_{\mathfrak{u}}, 1\right)=\frac{\partial^{|\mathfrak{u}|} F_{\mathfrak{u}}}{\partial \boldsymbol{y}_{\mathfrak{u}}}\left(\boldsymbol{y}_{\mathfrak{u}}\right) .
$$

The last equality holds because if there is an index $j$ such that $j \in \mathfrak{u}$ but $j \notin \mathfrak{u}^{\prime}$, then $F_{\mathfrak{u}^{\prime}}$ does not depend on $y_{j}$ and its partial derivative with respect to $y_{j}$ is 0 , while if there is an index $j$ such that $j \in \mathfrak{u}^{\prime}$ but $j \notin \mathfrak{u}$, then fixing $y_{j}$ at 1 annihilates $F_{\mathfrak{u}^{\prime}}$. It then follows that the norm in $\mathcal{W}_{s, \gamma}^{q, r}$ can also be expressed, for $1 \leq q \leq \infty$ and $1 \leq r<\infty$, in either of the forms

$$
\|F\|_{\mathcal{W}_{s, \gamma}^{q, r}}=\left(\sum_{\mathfrak{u} \subseteq\{1: s\}}\left\|\gamma_{\mathfrak{u}}^{-1 / 2} \frac{\partial^{|\mathfrak{u}|} F_{\mathfrak{u}}}{\partial \boldsymbol{y}_{\mathfrak{u}}}\left(\boldsymbol{y}_{\mathfrak{u}}\right)\right\|_{L^{q}}^{r}\right)^{1 / r}=\left(\sum_{\mathfrak{u} \subseteq\{1: s\}}\left\|F_{\mathfrak{u}}\right\|_{\mathcal{W}_{s, \gamma}^{q, r}}^{r}\right)^{1 / r},
$$

with the obvious modification for $r=\infty$. Hence we have a decomposition of the norm corresponding to the anchored decomposition. (Note that there needs to be 
a precise match between the chosen norm and the type of decomposition. For example, the unanchored variant of the norm should be combined with the ANOVA decomposition.)

It is generally too costly or even infeasible to compute the anchored decomposition of a given function $F$; however, the concept of an anchored decomposition is useful as a technical tool in QMC error analysis.

3.7. Extension of RKHS analysis to a Banach space setting As argued by Hickernell (see [13] or [14, Section 3.2]), we can often extend by continuity the inner product $\langle\cdot, \cdot\rangle_{\mathcal{W}}$ defined on $\mathcal{W} \times \mathcal{W}$ to $\mathcal{H} \times \mathcal{J}$, where $\mathcal{H} \supset \mathcal{W}$ and $\mathcal{J} \subset \mathcal{W}$. This allows the RKHS machinery with a given reproducing kernel to be extended to Banach spaces. More specifically, we have from (2.2) and the definition of the inner product (2.3) that

$$
\begin{aligned}
I_{S}(F)-Q_{s, N}(F) & =\left\langle F, \xi_{s, N}\right\rangle_{W_{s, \gamma}^{2,2}} \\
& =\sum_{\mathfrak{u} \subseteq\{1: s\}} \gamma_{\mathfrak{u}}^{-1} \int_{[0,1]^{|\mathfrak{u}|}} \frac{\partial^{|\mathfrak{u}|} F}{\partial \boldsymbol{y}_{\mathfrak{u}}}\left(\boldsymbol{y}_{\mathfrak{u}}, 1\right) \frac{\partial^{|\mathfrak{u}|} \xi_{s, N}}{\partial \boldsymbol{y}_{\mathfrak{u}}}\left(\boldsymbol{y}_{\mathfrak{u}}, 1\right) d \boldsymbol{y}_{\mathfrak{u}} .
\end{aligned}
$$

For any $\boldsymbol{y} \in[0,1]^{s}$, the kernel $K_{s, \gamma}(\cdot, \boldsymbol{y})$ given by (2.4) has sufficient smoothness to lie not only in $\mathcal{W}_{s, \gamma}^{2,2}$ but also in $\mathcal{W}_{s, \gamma}^{\infty, 1}$, and thus the reproducing property of the kernel holds for all $F \in \mathcal{W}_{s, \gamma}^{q, r}$ and for all $q$ and $r$. Likewise, $\xi_{s, N} \in \mathcal{W}_{s, \gamma}^{\infty, 1}$, and so the above equality holds for all $F \in \mathcal{W}_{s, \gamma}^{q, r}$ and for all $q$ and $r$.

Introducing weights $\gamma_{\mathfrak{u}}^{1 / 2}$ and applying Hölder's inequality twice with conjugate pairs $q, q^{\prime}$ and $r, r^{\prime}$ in the same manner as in Section 3.1, we obtain another form of the weighted Koksma-Hlawka inequality:

$$
\left|I_{S}(F)-Q_{s, N}(F)\right| \leq\|F\|_{\mathcal{W}_{s, r}^{q, r}}\left(\sum_{\mathfrak{u} \subseteq\{1: s\}}\left\|\gamma_{\mathfrak{u}}^{1 / 2} \frac{\partial^{|\mathfrak{u}|} \xi_{s, N}}{\partial \boldsymbol{y}_{\mathfrak{u}}}\left(\boldsymbol{y}_{\mathfrak{u}}, 1\right)\right\|_{L^{q^{\prime}}}^{r^{\prime}}\right)^{1 / r^{\prime}} .
$$

It can be verified that the second factor on the right-hand side is precisely the worstcase error $e^{\text {wor }}\left(\mathcal{P} ; \mathcal{W}_{s, \gamma}^{q, r}\right)$, which is exactly the weighted discrepancy $D_{s, \gamma}^{q^{\prime}, r^{\prime}}(\mathcal{P})$.

This approach can be useful when we are given a reproducing kernel and cannot use the Hlawka-Zaremba identity.

3.8. Relation between discrepancies with different $\boldsymbol{r}$ We recall from (3.8) that for fixed $1 \leq q^{\prime} \leq \infty$,

$$
D_{s, \gamma}^{q^{\prime}, r_{1}^{\prime}}(\mathcal{P}) \geq D_{s, \gamma}^{q^{\prime}, r_{2}^{\prime}}(\mathcal{P}) \quad \text { for all } 1 \leq r_{1}^{\prime}<r_{2}^{\prime} \leq \infty
$$

We now give two lemmas relating these discrepancies in other ways by modifying the weights.

The first lemma provides a result in the opposite direction to the inequality above. It allows us to use known results for $r=1,2$ to draw conclusions for other values of $r$. 
Lemma 3.2. Let $1 \leq q^{\prime} \leq \infty$ and $1 \leq r_{1}^{\prime}<r_{2}^{\prime} \leq \infty$. Define new weights

$$
\widetilde{\gamma}_{\mathfrak{u}}:=\gamma_{\mathfrak{u}}^{a}, \quad \mathfrak{u} \subseteq\{1: s\}
$$

for arbitrary $a \in[0,1]$. Then

$$
D_{s, \gamma}^{q^{\prime}, r_{1}^{\prime}}(\mathcal{P}) \leq\left(\sum_{\emptyset \neq u \subseteq \subseteq 1: s\}} \gamma_{\mathfrak{u}}^{(1-a) r_{1}^{\prime} r_{2}^{\prime} /\left(2\left(r_{2}^{\prime}-r_{1}^{\prime}\right)\right)}\right)^{\left(r_{2}^{\prime}-r_{1}^{\prime}\right) /\left(r_{1}^{\prime} r_{2}^{\prime}\right)} D_{s, \bar{\gamma}}^{q^{\prime}, r_{2}^{\prime}}(\mathcal{P}),
$$

with the convention that for $r_{2}^{\prime}=\infty$ we have $\left(r_{2}^{\prime}-r_{1}^{\prime}\right) /\left(r_{1}^{\prime} r_{2}^{\prime}\right)=1 / r_{1}^{\prime}$.

Proof. This result was proved by Hickernell et al. [17, Lemma 1] by using a relationship between the corresponding norms. Here we use a similar argument to prove the result directly:

$$
\begin{aligned}
& D_{s, \gamma}^{q^{\prime}, r_{1}^{\prime}}(\mathcal{P})=\left(\sum_{\emptyset \neq u \subseteq\{1: s\}}\left\|\gamma_{\mathfrak{u}}^{1 / 2} \operatorname{discr} \mathcal{P}\left(\boldsymbol{y}_{\mathfrak{u}}, 1\right)\right\|_{L^{q^{\prime}}}\right)^{r_{1}^{\prime}} \\
& =\left(\sum_{\emptyset \neq \mathfrak{u} \subseteq 1: s\}} \gamma_{\mathfrak{u}}^{(1-a) r_{1}^{\prime} / 2}\left\|\gamma_{\mathfrak{u}}^{a / 2} \operatorname{discr} \mathcal{P}\left(\boldsymbol{y}_{\mathfrak{u}}, 1\right)\right\|_{L^{q^{\prime}}}^{r_{1}^{\prime}}\right)^{1 / r_{1}^{\prime}} \\
& \leq\left(\sum_{\emptyset \neq u \subseteq \subseteq 1: s\}} \gamma_{\mathfrak{u}}^{(1-a) r_{1}^{\prime} t / 2}\right)^{1 /\left(r_{1}^{\prime} t\right)}\left(\sum_{\emptyset \neq u \subseteq \subseteq\{1: s\}}\left\|\gamma_{\mathfrak{u}}^{a / 2} \operatorname{discr} \mathcal{P}\left(\boldsymbol{y}_{\mathfrak{u}}, 1\right)\right\|_{L^{q^{\prime}}}\right)^{r_{2}^{\prime}} \text {, }
\end{aligned}
$$

where we apply Hölder's inequality with $t$ being the Hölder conjugate of $r_{2}^{\prime} / r_{1}^{\prime}$, that is, $t=1 /\left(1-r_{1}^{\prime} / r_{2}^{\prime}\right)=r_{2}^{\prime} /\left(r_{2}^{\prime}-r_{1}^{\prime}\right)$.

The second lemma allows us to trade a lower convergence rate for the discrepancy in return for less restrictive conditions on the weights.

Lemma 3.3. Let $1 \leq q^{\prime} \leq \infty$ and $1 \leq r_{1}^{\prime} \leq r_{2}^{\prime}<\infty$. Define new weights

$$
\widetilde{\gamma}_{\mathrm{u}}:=\gamma_{\mathrm{u}}^{r_{\mathrm{u}}^{\prime}} / r_{1}^{\prime} \text {. }
$$

Then

$$
D_{s, \gamma}^{q^{\prime}, r_{2}^{\prime}}(\mathcal{P}) \leq\left[D_{s, \tilde{\gamma}}^{q^{\prime}, r_{1}^{\prime}}(\mathcal{P})\right]^{r_{1}^{\prime} / r_{2}^{\prime}}
$$

Proof. This result was proved by Hickernell et al. [19, Lemma 1] for a special discrepancy, but the same argument holds here:

$$
\begin{aligned}
D_{s, \gamma}^{q^{\prime}, r_{2}^{\prime}}(\mathcal{P}) & =\left(\sum_{\emptyset \neq u \subseteq\{1: s\}}\left\|\gamma_{\mathfrak{u}}^{1 / 2} \operatorname{discr} \mathcal{P}\left(\boldsymbol{y}_{\mathfrak{u}}, 1\right)\right\|_{L^{q^{\prime}}}^{r_{2}^{\prime}}\right)^{1 / r_{2}^{\prime}} \\
& \leq\left(\sum_{\emptyset \neq \mathfrak{u} \subseteq\{1: s\}}\left\|\widehat{\gamma}_{\mathfrak{u}}^{1 / 2} \operatorname{discr} \mathcal{P}\left(\boldsymbol{y}_{\mathfrak{u}}, 1\right)\right\|_{L^{q^{\prime}}}^{r_{1}^{\prime}}\right)^{1 / r_{2}^{\prime}}=\left[D_{s, \bar{\gamma}}^{q^{\prime}, r_{1}^{\prime}}(\mathcal{P})\right]^{r_{1}^{\prime} / r_{2}^{\prime}},
\end{aligned}
$$

where we have used the estimate $\left\|\operatorname{discr} \mathcal{P}\left(\boldsymbol{y}_{\mathfrak{u}}, 1\right)\right\|_{L^{q^{\prime}}}^{r_{2}^{\prime} / r_{1}^{\prime}} \leq\left\|\operatorname{discr} \mathcal{P}\left(\boldsymbol{y}_{\mathfrak{u}}, 1\right)\right\|_{L^{q^{\prime}}}$ following from the fact that $r_{2}^{\prime} / r_{1}^{\prime} \geq 1$ and $|\operatorname{discr} \mathcal{P}(\boldsymbol{y})| \leq 1$ for all $\boldsymbol{y} \in[0,1]^{s}$. 
3.9. Tractability Tractability of multivariate problems has attracted much attention recently. For the full story, see the books by Novak and Woźniakowski [40, 41]. Here we only briefly discuss the general concept.

Roughly speaking, the integration problem is tractable in a function space if there exists a quadrature rule whose worst-case error is bounded polynomially in $N^{-1}$ and $s$, with an implied constant that is independent of both $N$ and $s$; it is said to be strongly tractable if the bound is independent of $s$.

Tractability for the standard setting, namely the Hilbert space $\mathcal{W}_{s, \gamma}^{2,2}=\mathcal{H}_{s, \gamma}$ with product weights, was analysed by Novak and Woźniakowski [39], who proved, for all possible algorithms that make use of at most $N$ point evaluations of $F$, that (1.9) is a necessary and sufficient condition on the weights to achieve strong tractability.

For the Banach spaces $\mathcal{W}_{s, \gamma}^{q, r}$ with product weights, it was proved by Hickernell et al. [16-19] (for QMC algorithms only) that a necessary and sufficient condition for strong tractability is essentially

$$
\sum_{j=1}^{\infty} \gamma_{j}^{r^{\prime} / 2}<\infty .
$$

(There are some exceptions: the necessity of condition (3.9) for $q=1$ does not seem to be known, and for $q<2$ the exponent $r^{\prime} / 2$ in the sufficiency of condition (3.9) should be replaced by $r^{\prime} / 2-\delta$ for $\delta>0$ arbitrarily small.) The main observation here is that the tractability conditions are determined by the value of $r$ alone. This is another reason to support the decoupling of $q$ and $r$.

Note that the sufficiency of condition (3.9) was obtained by Hickernell et al. [17] by relating all discrepancies to $D_{s, \gamma}^{\infty, \infty}(\mathcal{P})$ or $D_{s, \gamma}^{2,2}(\mathcal{P})$ using Lemma 3.2 and then applying nonconstructive results. Constructive results generally require stronger assumptions on the weights; we discuss these in Section 4.

3.10. Infinite-dimensional integration Integration in the infinite-dimensional setting has been analysed in a number of recent papers [10, 15, 34, 38, 44], mostly in a Hilbert space setting. In a formal sense, there is little difficulty in considering integration with an infinite number of variables; for a function $F$ of infinitely many variables $\boldsymbol{y}=\left(y_{1}, y_{2}, \ldots\right)$, we define the integral of $F$ as

$$
I(F)=\lim _{s \rightarrow \infty} \int_{[0,1]^{s}} F\left(y_{1}, \ldots, y_{s}, a, a, \ldots\right) d\left(y_{1}, \ldots, y_{s}\right)
$$

whenever this limit exists, for some fixed anchor $a \in[0,1]$. Moreover, the QMC approximation is always applied to $F\left(y_{1}, \ldots, y_{s}, a, a, \ldots\right)$ for a suitable $s$, and thus we only ever need to evaluate functions with a finite number of variables different from the anchor $a$. But an additional level of error analysis is needed to handle the truncation to a finite number of variables different from $a$, and a new question presents itself, namely: how should the cost of evaluating $F\left(y_{1}, \ldots, y_{s}, a, a, \ldots\right)$ depend on $s$ ? The papers cited above have obtained results relating to the last question under various hypotheses, with the only common agreement being that the cost should increase with $s$. The model should of course depend on the problem at hand. 
Note that the probabilistic example in Section 1.5 has a natural extension to infinite dimensions: all we need to do is let the sum in (1.16) go from 1 to $\infty$ instead of from 1 to $s$. The problem considered in that section is then obtained by choosing the anchor to be $a=0$ and setting all variables $y_{j}$ with $j \geq s+1$ to the value 0 , and the infinite-dimensional version corresponds to letting $s \rightarrow \infty$.

\section{Constructive QMC methods}

4.1. CBC construction of lattice rules based on "shift-averaged" worst-case error Here we describe the construction of randomly shifted lattice rules in the Hilbert space $\mathcal{W}_{s, \gamma}^{2,2}$. We denote by $D_{s, \gamma}^{2,2}(z, \Delta)$ the worst-case error (or discrepancy) of a shifted lattice rule in $\mathcal{W}_{s, \gamma}^{2,2}$; an explicit formula can be obtained from (3.7). Since we use random shifts, the criterion we use for the CBC construction of the generating vector $z$ is the shift-averaged worst-case error $e_{N, s, \gamma}(z)$, defined by

$$
e_{N, s, \gamma}^{2}(z):=\int_{\Delta \in[0,1]^{s}}\left[D_{s, \gamma}^{2,2}(z, \Delta)\right]^{2} d \Delta .
$$

Using term-by-term integration of (3.7), it can be shown that

$$
e_{N, s, \gamma}^{2}(z)=\sum_{\emptyset \neq \mathfrak{u} \subseteq\{1: s\}} \gamma_{\mathfrak{u}}\left(\frac{1}{N} \sum_{k=0}^{N-1} \prod_{j \in \mathfrak{u}}\left[B_{2}\left(\left\{\frac{k z_{j}}{N}\right\}\right)+m\right]-m^{|\mathfrak{u}|}\right),
$$

where the braces again indicate taking the fractional part of a real number, $B_{2}(x):=$ $x^{2}-x+1 / 6$ is the Bernoulli polynomial of degree two, and $m=1 / 3$ in the current space with anchor 1 . In general, $m=a^{2}-a+1 / 3$ for a general anchor $a$, and $m=0$ for the unanchored variant. (Note that the sum now runs from 0 to $N-1$, which is allowable because the $k=0$ and $k=N$ terms are equal.)

Starting with $z_{1}=1$, for each $d=2,3, \ldots, s$ the CBC algorithm chooses $z_{d}$ to be the value from the set $U_{N}:=\{1 \leq u \leq N-1: \operatorname{gcd}(u, N)=1\}$ that minimizes $e_{N, d, \gamma}^{2}\left(z_{1}, \ldots, z_{d-1}, z_{d}\right)$, with the previously chosen components $z_{1}, \ldots, z_{d-1}$ held fixed. The total number of choices in each dimension is given by the Euler totient function

$$
\phi(N)=\left|U_{N}\right|=|\{1 \leq u \leq N-1: \operatorname{gcd}(u, N)=1\}| .
$$

The computational cost of the $\mathrm{CBC}$ construction with general weights is exponential in $s$, but it can be as low as $\mathrm{O}(s N \ln N)$ operations for some special forms of weights, including POD weights; this is discussed in Section 5. The following theorem provides the theoretical justification for the CBC construction.

THEOREM 4.1. The generating vector $z$ constructed by the CBC algorithm, minimizing $e_{N, s, \gamma}^{2}(z)$ in each step, satisfies, for any $\lambda \in(1 / 2,1]$,

$$
e_{N, s, \gamma}^{2}(z) \leq\left(\sum_{\emptyset \neq \mathfrak{u} \subseteq\{1: s\}} \gamma_{\mathfrak{u}}^{\lambda}(\rho(\lambda))^{\mid \mathfrak{u l |}}\right)^{1 / \lambda}[\phi(N)]^{-1 / \lambda}
$$


with

$$
\rho(\lambda):=\frac{2 \zeta(2 \lambda)}{\left(2 \pi^{2}\right)^{\lambda}}+m^{\lambda}
$$

where $\zeta(x)$ is the Riemann zeta function as in Section 1.5 and $\phi(N)$ is the Euler totient function given by (4.2).

Proof. This result is partially derived by Sloan et al. [52, Theorem 3(A)] for prime $N$ by exploiting the connection between the Sobolev and Korobov spaces. Rather than building on this derivation, here we provide a direct proof for general $N$.

The Bernoulli polynomial has the expansion $B_{2}(x)=\sum_{h \neq 0} e^{2 \pi i h x} /\left(2 \pi^{2} h^{2}\right)$. Thus the constant $m$ inside the product in (4.1) can be interpreted as the $h=0$ term. A crucial technical step, if an argument later in this proof is to work, is to remove this $h=0$ term from the product. We proceed as follows. Upon writing $b_{j}:=B_{2}\left(\left\{k z_{j} / N\right\}\right)$ and adopting the convention that an empty product is 1 , we obtain

$$
\begin{aligned}
e_{N, s, \gamma}^{2}(z) & =\frac{1}{N} \sum_{k=0}^{N-1} \sum_{\mathfrak{u} \subseteq\{1: s\}} \gamma_{\mathfrak{u}} \prod_{j \in \mathfrak{u}}\left(b_{j}+m\right)-\sum_{\mathfrak{u} \subseteq\{1: s\}} \gamma_{\mathfrak{u}} m^{|\mathfrak{u}|} \\
& =\frac{1}{N} \sum_{k=0}^{N-1} \sum_{\mathfrak{u} \subseteq\{1: s\}} \gamma_{\mathfrak{u}} \sum_{\mathfrak{v} \subseteq \mathfrak{u}} m^{|\mathfrak{u}|-|\mathfrak{v}|} \prod_{j \in \mathfrak{v}} b_{j}-\sum_{\mathfrak{u} \subseteq\{1: s\}} \gamma_{\mathfrak{u}} m^{|\mathfrak{u}|} \\
& =\frac{1}{N} \sum_{k=0}^{N-1} \sum_{\mathfrak{v} \subseteq\{1: s\}} \sum_{\mathfrak{v} \subseteq \mathfrak{u} \subseteq\{1: s\}} \gamma_{\mathfrak{u}} m^{|\mathfrak{u}|-|\mathfrak{v}|} \prod_{j \in \mathfrak{v}} b_{j}-\sum_{\mathfrak{u} \subseteq\{1: s\}} \gamma_{\mathfrak{u}} m^{|\mathfrak{u}|} \\
& =\frac{1}{N} \sum_{k=0}^{N-1} \sum_{\mathfrak{v} \subseteq\{1: s\}} \tilde{\gamma}_{\mathfrak{v}} \prod_{j \in \mathfrak{v}} b_{j}-\tilde{\gamma}_{\emptyset} \\
& =\sum_{\emptyset \neq \mathfrak{v} \subseteq\{1: s\}} \tilde{\gamma}_{\mathfrak{v}}\left(\frac{1}{N} \sum_{k=0}^{N-1} \prod_{j \in \mathfrak{v}} b_{j}\right),
\end{aligned}
$$

where we have introduced auxiliary weights defined by

$$
\tilde{\gamma}_{\mathfrak{v}}:=\sum_{\mathfrak{v} \subseteq \mathfrak{u} \subseteq\{1: s\}} \gamma_{\mathfrak{u}} m^{|\mathfrak{u}|-|\mathfrak{p}|}, \quad \mathfrak{v} \subseteq\{1: s\} .
$$

Next, we prove that the CBC construction yields

$$
e_{N, s, \gamma}^{2}(z) \leq\left(\sum_{\emptyset \neq \mathfrak{v} \subseteq\{1: s\}} \tilde{\gamma}_{\mathfrak{v}}^{\lambda}\left(\frac{2 \zeta(2 \lambda)}{\left(2 \pi^{2}\right)^{\lambda}}\right)^{|\mathfrak{p}|}\right)^{1 / \lambda}[\phi(N)]^{-1 / \lambda}
$$

for all $\lambda \in(1 / 2,1]$. This is proved by induction on $s$. The base step $s=1$ is straightforward to verify, and we omit the details here. Assume now that we have chosen the first $s-1$ components $z_{1}, \ldots, z_{s-1}$ and that (4.4) holds with $s$ 
replaced by $s-1$. Using the expansion of $B_{2}$ and the "character property" of lattice rules (namely that $(1 / N) \sum_{k=0}^{N-1} e^{2 \pi \mathrm{i} k \boldsymbol{h} \cdot z / N}$ is 1 if $\boldsymbol{h} \cdot \boldsymbol{z} \equiv 0(\bmod N)$ and 0 otherwise), we write

$$
\begin{aligned}
e_{N, s, \gamma}^{2}(\boldsymbol{z}) & =\sum_{\emptyset \neq \mathfrak{v} \subseteq\{1: s\}} \tilde{\gamma}_{\mathfrak{v}}\left(\frac{1}{N} \sum_{k=0}^{N-1} \prod_{j \in \mathfrak{v}} \sum_{h \neq 0} \frac{e^{2 \pi \mathrm{i} h k z_{j} / N}}{2 \pi^{2} h^{2}}\right) \\
& =\sum_{\emptyset \neq \mathfrak{v} \subseteq\{1: s\}} \frac{\tilde{\gamma}_{\mathfrak{v}}}{\left(2 \pi^{2}\right)^{|\mathfrak{p}|}}\left(\frac{1}{N} \sum_{k=0}^{N-1} \sum_{\boldsymbol{h}_{\mathfrak{v}} \in(\mathbb{Z} \backslash\{0\})^{|\mathfrak{p}|}} \frac{e^{2 \pi \mathrm{i} k \boldsymbol{h}_{\mathfrak{v}} \cdot z_{\mathfrak{v}} / N}}{\prod_{j \in \mathfrak{v}} h_{j}^{2}}\right) \\
& =\sum_{\emptyset \neq \mathfrak{v} \subseteq\{1: s\}} \frac{\tilde{\gamma}_{\mathfrak{v}}}{\left(2 \pi^{2}\right)^{|\mathfrak{p}|}}\left(\sum_{\substack{\boldsymbol{h}_{\mathfrak{v}} \in(\mathbb{Z} \backslash\{0\})^{|\mathfrak{p}|} \\
\boldsymbol{h}_{\mathfrak{v}} \cdot z_{\mathfrak{v}} \equiv 0(\bmod N)}} \frac{1}{\prod_{j \in \mathfrak{v}} h_{j}^{2}}\right) .
\end{aligned}
$$

Now we separate the terms according to whether or not the element $s$ is included in the set $\mathfrak{v}$, obtaining the recursive expression

$$
e_{N, s, \gamma}^{2}\left(z_{1}, \ldots, z_{s-1}, z_{s}\right)=e_{N, s-1, \gamma}^{2}\left(z_{1}, \ldots, z_{s-1}\right)+\theta\left(z_{s}\right),
$$

where (suppressing the dependence of $\theta$ on $z_{1}, \ldots, z_{s-1}$ )

$$
\theta\left(z_{s}\right):=\sum_{s \in \mathfrak{v} \subseteq\{1: s\}} \frac{\tilde{\gamma}_{\mathfrak{v}}}{\left(2 \pi^{2}\right)^{|\mathfrak{p}|}}\left(\sum_{h_{s} \in \mathbb{Z} \backslash\{0\}} \frac{1}{h_{s}^{2}} \sum_{\substack{\boldsymbol{h}_{\mathfrak{v} \backslash\langle s\}} \in(\mathbb{Z} \backslash\{0\})^{|\mathfrak{p}|-1} \\ \boldsymbol{h}_{\mathfrak{v} \backslash\langle s\}} z_{\mathfrak{v} \backslash\langle\{s\}} \equiv-h_{s} z_{s}(\bmod N)}} \frac{1}{\prod_{j \in \mathfrak{v} \backslash\{s\}} h_{j}^{2}}\right) .
$$

If $z_{s}^{*}$ denotes the value chosen by the $\mathrm{CBC}$ algorithm in dimension $s$, then (since the minimum is always smaller than or equal to the average) we have, for all $\lambda \in(0,1]$,

$$
\begin{aligned}
& {\left[\theta\left(z_{s}^{*}\right)\right]^{\lambda} \leq \frac{1}{\phi(N)} \sum_{z_{s} \in U_{N}}\left[\theta\left(z_{s}\right)\right]^{\lambda}} \\
& \leq \frac{1}{\phi(N)} \sum_{z_{s} \in U_{N}} \sum_{s \in \mathfrak{v} \subseteq\{1: s\}} \frac{\tilde{\gamma}_{\mathfrak{v}}^{\lambda}}{\left(2 \pi^{2}\right)^{|\mathfrak{p}| \lambda}} \\
& \times\left(\sum_{h_{s} \in \mathbb{Z} \backslash\{0\}} \frac{1}{\left|h_{s}\right|^{2 \lambda}} \sum_{\substack{h_{\mathfrak{v} \backslash\langle s s} \in(\mathbb{Z} \backslash\{0\})^{|\mathfrak{p}|-1} \\
\boldsymbol{h}_{\mathfrak{p} \backslash\langle s\}} z_{\mathfrak{p} \backslash\langle\langle s|} \equiv-h_{s} z_{s}(\bmod N)}} \frac{1}{\prod_{j \in \mathfrak{v} \backslash\{s\}}\left|h_{j}\right|^{2 \lambda}}\right),
\end{aligned}
$$

where we have used the inequality (sometimes mistakenly referred to as Jensen's inequality)

$$
\left(\sum_{k} a_{k}\right)^{\lambda} \leq \sum_{k} a_{k}^{\lambda}, \quad a_{k} \geq 0, \lambda \in(0,1]
$$


Next, we separate the terms according to whether or not $h_{s}$ is a multiple of $N$, and obtain

$$
\begin{aligned}
& {\left[\theta\left(z_{s}^{*}\right)\right]^{\lambda}} \\
& \leq \sum_{s \in \mathfrak{v} \subseteq\{1: s\}} \frac{\tilde{\gamma}_{\mathfrak{v}}^{\lambda}}{\left(2 \pi^{2}\right)^{|\mathfrak{p}| \lambda}} \cdot \frac{2 \zeta(2 \lambda)}{N^{2 \lambda}}\left(\sum_{\substack{\boldsymbol{h}_{\backslash \backslash s s} \in(\mathbb{Z} \backslash\{0\})^{\mathfrak{p} \mid-1} \\
\boldsymbol{h}_{\mathfrak{v} \backslash\langle s\rangle} \cdot z_{\mathfrak{v} \backslash\langle s\}} \equiv 0(\bmod N)}} \frac{1}{\prod_{j \in \mathfrak{v} \backslash\{s\}}\left|h_{j}\right|^{2 \lambda}}\right)
\end{aligned}
$$

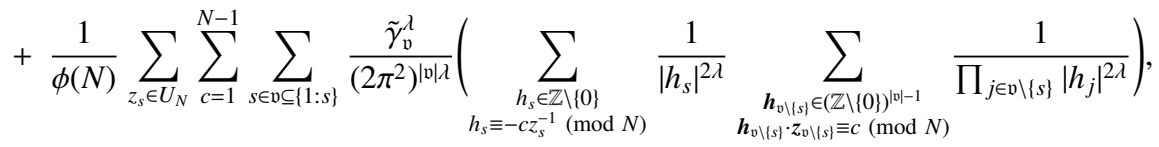

where $z_{s}^{-1}$ is the multiplicative inverse of $z_{s}$ in $U_{N}$, such that $z_{s} z_{s}^{-1} \equiv 1(\bmod N)$. For fixed $c$ satisfying $1 \leq c \leq N-1$, we have $\left\{c z_{s}^{-1}(\bmod N): z_{s} \in U_{N}\right\}=\{c z(\bmod N)$ : $\left.z \in U_{N}\right\}$. Let $g=\operatorname{gcd}(c, N)$. Then $\operatorname{gcd}(c / g, N / g)=1$, and

$$
\begin{aligned}
& \sum_{z_{s} \in U_{N}} \sum_{\substack{h_{s} \in \mathbb{Z} \backslash\{0\} \\
h_{s} \equiv-c z_{s}^{-1}(\bmod N)}} \frac{1}{\left|h_{s}\right|^{2 \lambda}}=\sum_{z \in U_{N}} \sum_{\substack{h_{s} \in \mathbb{Z} \backslash\{0\} \\
h_{s} \equiv-c z(\bmod N)}} \frac{1}{\left|h_{s}\right|^{2 \lambda}} \\
& =\sum_{z \in U_{N}} \sum_{m \in \mathbb{Z}} \frac{1}{|m N-c z|^{2 \lambda}} \\
& =g^{-2 \lambda} \sum_{z \in U_{N}} \sum_{m \in \mathbb{Z}} \frac{1}{|m(N / g)-(c / g) z|^{2 \lambda}} \\
& =g^{-2 \lambda} \sum_{z \in U_{N}} \sum_{\substack{h \in \mathbb{Z} \backslash\{0\} \\
h \equiv-(c / g) z(\bmod N / g)}} \frac{1}{|h|^{2 \lambda}} \\
& \leq g^{-2 \lambda} g \sum_{a=1}^{N / g-1} \sum_{\substack{h \in \mathbb{Z} \backslash\{0\} \\
h \equiv a(\bmod N / g)}} \frac{1}{|h|^{2 \lambda}} \\
& =g^{1-2 \lambda} \cdot 2 \zeta(2 \lambda)\left(1-(N / g)^{-2 \lambda}\right) \\
& \leq 2 \zeta(2 \lambda) \text {, }
\end{aligned}
$$

with the last step following because $g \geq 1$ and $\lambda>1 / 2$. The condition $\lambda>1 / 2$ is needed to ensure that $\zeta(2 \lambda)<\infty$. Hence

$$
\begin{aligned}
& {\left[\theta\left(z_{s}^{*}\right)\right]^{\lambda} \leq \sum_{s \in \mathfrak{v} \subseteq\{1: s\}} \frac{\tilde{\gamma}_{\mathfrak{v}}^{\lambda}}{\left(2 \pi^{2}\right)^{|\mathfrak{p}| \lambda}} \cdot \frac{2 \zeta(2 \lambda)}{N^{2 \lambda}}\left(\sum_{\substack{\boldsymbol{h}_{\mathfrak{v} \backslash \backslash s\}} \in(\mathbb{Z} \backslash\{0\})^{|\mathfrak{p}|-1} \\
\boldsymbol{h}_{\mathfrak{v} \backslash\langle s\}} \cdot z_{\mathfrak{p} \backslash\langle s\}} \equiv 0(\bmod N)}} \frac{1}{\prod_{j \in \mathfrak{v} \backslash\{s\}}\left|h_{j}\right|^{2 \lambda}}\right)} \\
& +\frac{1}{\phi(N)} \sum_{s \in \mathfrak{v} \subseteq\{1: s\}} \frac{\tilde{\gamma}_{\mathfrak{v}}^{\lambda}}{\left(2 \pi^{2}\right)^{|\mathfrak{p}| \lambda}} \cdot 2 \zeta(2 \lambda)\left(\sum_{\substack{\boldsymbol{h}_{\mathfrak{v} \backslash\langle s\}} \in(\mathbb{Z} \backslash\{0\}\}^{\mid \mathfrak{p}-1} \\
\boldsymbol{h}_{\mathfrak{v} \backslash\langle s\}} \cdot \mathbb{Z}_{\mathfrak{v} \backslash\langle s|} \neq 0(\bmod N)}} \frac{1}{\prod_{j \in \mathfrak{v} \backslash\{s\}}\left|h_{j}\right|^{2 \lambda}}\right) \\
& \leq \frac{1}{\phi(N)} \sum_{s \in \mathfrak{v} \subseteq\{1: s\}} \tilde{\gamma}_{\mathfrak{v}}^{\lambda}\left(\frac{2 \zeta(2 \lambda)}{\left(2 \pi^{2}\right)^{\lambda}}\right)^{|\mathfrak{v}|}
\end{aligned}
$$

This, together with (4.5) and the induction hypothesis, yields the result (4.4). 
Finally, we express the result in terms of the original weights. Writing $\alpha_{\lambda}:=$ $2 \zeta(2 \lambda) /\left(2 \pi^{2}\right)^{\lambda}$ and using (4.3) and (4.6) gives

$$
\begin{aligned}
\sum_{\emptyset \neq \mathfrak{v} \subseteq\{1: s\}} \tilde{\gamma}_{\mathfrak{v}}^{\lambda} \alpha_{\lambda}^{|\mathfrak{v}|} & \leq \sum_{\mathfrak{v} \subseteq\{1: s\}} \sum_{\mathfrak{v} \subseteq \mathfrak{u} \subseteq\{1: s\}} \gamma_{\mathfrak{u}}^{\lambda} m^{(|\mathfrak{u}|-|\mathfrak{v}|) \lambda} \alpha_{\lambda}^{|\mathfrak{v}|}-\sum_{\mathfrak{u} \subseteq\{1: s\}} \gamma_{\mathfrak{u}}^{\lambda} m^{|\mathfrak{u}| \lambda} \\
& =\sum_{\mathfrak{u} \subseteq\{1: s\}} \gamma_{\mathfrak{u}}^{\lambda} \sum_{\mathfrak{v} \subseteq \mathfrak{u}} m^{(|\mathfrak{u}|-|\mathfrak{v}|) \lambda} \alpha_{\lambda}^{|\mathfrak{v}|}-\sum_{\mathfrak{u} \subseteq\{1: s\}} \gamma_{\mathfrak{u}}^{\lambda} m^{|\mathfrak{u}| \lambda} \\
& =\sum_{\mathfrak{u} \subseteq\{1: s\}} \gamma_{\mathfrak{u}}^{\lambda}\left(\alpha_{\lambda}+m^{\lambda}\right)^{|\mathfrak{u}|}-\sum_{\mathfrak{u} \subseteq\{1: s\}} \gamma_{\mathfrak{u}}^{\lambda} m^{|\mathfrak{u}| \lambda} \\
& \leq \sum_{\mathfrak{\emptyset} \neq \mathfrak{u} \subseteq\{1: s\}} \gamma_{\mathfrak{u}}^{\lambda}\left(\alpha_{\lambda}+m^{\lambda}\right)^{|\mathfrak{u}|}
\end{aligned}
$$

This completes the proof.

THEOREM 4.2. If the weights satisfy

$$
\sum_{|\mathfrak{u}|<\infty} \gamma_{\mathfrak{u}}^{\lambda}(\rho(\lambda))^{|\mathfrak{u}|}<\infty \quad \text { for some } \lambda \in(1 / 2,1],
$$

or if we have product weights satisfying

$$
\sum_{j=1}^{\infty} \gamma_{j}^{\lambda}<\infty \quad \text { for some } \lambda \in(1 / 2,1]
$$

then the randomly shifted lattice rule constructed by the CBC algorithm based on $e_{N, s, \gamma}^{2}(z)$ satisfies

$$
\sqrt{\mathbb{E}\left[D_{s, \gamma}^{2,2}(z, \cdot)\right]^{2}} \leq C[\phi(N)]^{-1 /(2 \lambda)},
$$

where $\mathbb{E}$ denotes the expectation with respect to the random shift which is uniformly distributed over $[0,1]^{s}$, and $C$ is independent of $s$ and $N$ but goes to infinity as $\lambda \rightarrow 1 / 2$.

Proof. The result for general weights follows directly from Theorem 4.1 and the definition of $e_{N, s, \gamma}^{2}(z)$. In the case of product weights, we can write the sum in (4.7) as $\sum_{|\mathfrak{u}|<\infty} \gamma_{\mathfrak{u}}^{\lambda}(\rho(\lambda))^{|\mathfrak{u}|}=\prod_{j=1}^{\infty}\left(1+\rho(\lambda) \gamma_{j}^{\lambda}\right)=\exp \left(\sum_{j=1}^{\infty} \ln \left(1+\rho(\lambda) \gamma_{j}^{\lambda}\right)\right)$. The condition (4.8) is then deduced from the property that $\ln (1+x) \leq x$ for all $x \geq 0$.

Since, for large values of $N, 1 / \phi(N)=\mathrm{O}\left(N^{-1} \ln \ln N\right)$, if (4.7) or (4.8) in Theorem 4.2 holds with $\lambda$ arbitrarily close to $1 / 2$, then we have the convergence rate $\mathrm{O}\left(N^{-1+\delta}\right)$ for arbitrary $\delta>0$, with the implied constant approaching infinity as $\delta \rightarrow 0$.

4.2. CBC construction of lattice rules based on weighted $\boldsymbol{R}$ There is another search criterion that can be used in the CBC construction of lattice rules: we refer to it as "weighted $R$ "; see (4.12) below. It arises from a discrepancy bound involving the classical star discrepancy. Joe [26] proved a bound on the weighted star 
discrepancy $D_{s, \gamma}^{\infty, \infty}(\mathcal{P})$ for such a CBC construction with product weights and prime $N$. This was subsequently extended to general weights and/or composite $N$ [45-47].

The search criterion used for general weights $[45,47]$ has a fundamental difference from the criterion used for product weights [26, 46]. The discrepancy bounds established by Joe and Sinescu $[26,46]$ can be applied to all discrepancies $D_{s, \gamma}^{q^{\prime}, r^{\prime}}(\mathcal{P})$, but the same is not true for the results in the other two aforementioned papers [45, 47]. Moreover, the results in those papers [45, 47] rely on a (restrictive) monotonicity assumption on the weights:

$$
\gamma_{\mathfrak{v}} \geq \gamma_{\mathfrak{u}} \quad \text { whenever } \mathfrak{v} \subseteq \mathfrak{u} .
$$

In particular, this condition does not hold for POD weights.

Here we present a discrepancy bound for general weights, which (i) uses a direct extension of the search criterion from the papers of Joe and Sinescu [26, 46] for product weights, (ii) applies to all discrepancies $D_{s, \gamma}^{q^{\prime}, r^{\prime}}(\mathcal{P})$, and (iii) does not require the monotonicity assumption (4.9). The results obtained in this subsection are new.

Recall that for all $q^{\prime}, r^{\prime} \geq 1$,

$$
D_{s, \gamma}^{q^{\prime}, r^{\prime}}(\mathcal{P}) \leq D_{s, \gamma}^{\infty, 1}(\mathcal{P})=\sum_{\emptyset \neq u \subseteq\{1: s\}} \gamma_{\mathfrak{u}}^{1 / 2}\left[D^{*}\left(\mathcal{P}_{\mathfrak{u}}\right)\right]
$$

where $D^{*}\left(\mathcal{P}_{\mathfrak{u}}\right)$ is the classical star discrepancy of $\mathcal{P}_{\mathfrak{u}}$, the projection of the point set $\mathcal{P}$ in the coordinates $\boldsymbol{y}_{\mathfrak{u}}$. When $\mathcal{P}$ is a lattice rule with generating vector $z$, it is known [36, Theorems 3.10 and 5.6] that

$$
D^{*}\left(\mathcal{P}_{\mathfrak{u}}\right) \leq 1-\left(1-\frac{1}{N}\right)^{|\mathfrak{u}|}+\frac{R_{N}\left(z_{\mathfrak{u}}\right)}{2},
$$

where

$$
R_{N}\left(z_{\mathfrak{u l}}\right):=\frac{1}{N} \sum_{k=0}^{N-1} \prod_{j \in \mathfrak{u}}\left(1+\sum_{\substack{-N / 2<h \leq N / 2 \\ h \neq 0}} \frac{e^{2 \pi \mathrm{i} h k z_{j} / N}}{|h|}\right)-1 .
$$

Hence we conclude that for all $q^{\prime}, r^{\prime} \geq 1$,

$$
D_{s, \gamma}^{q^{\prime}, r^{\prime}}(\mathcal{P}) \leq \sum_{\emptyset \neq \mathfrak{u} \subseteq\{1: s\}} \gamma_{\mathfrak{u}}^{1 / 2}\left(1-\left(1-\frac{1}{N}\right)^{|\mathfrak{u}|}\right)+\frac{R_{N, s, \gamma}(z)}{2}
$$

where

$$
R_{N, s, \gamma}(z):=\sum_{\emptyset \neq \mathfrak{u} \subseteq\{1: s\}} \gamma_{\mathfrak{u}}^{1 / 2} R_{N}\left(z_{\mathfrak{u}}\right) .
$$

THEOREM 4.3. The generating vector $z$ constructed by the CBC algorithm, minimizing $R_{N, s, \gamma}(z)$ in each step, satisfies

$$
R_{N, s, \gamma}(z) \leq \frac{2}{N} \sum_{\emptyset \neq u \in\{1: s\}} \gamma_{\mathfrak{u}}^{1 / 2}\left(c_{\text {lat }} \ln N\right)^{|\mathfrak{u}|},
$$


where

$$
c_{\text {lat }}:=\sup _{N \geq 2}\left\{\frac{1}{\ln N}+2+\frac{2 \pi^{2}(N-1)}{3 \phi(N) \ln N}\right\} .
$$

Proof. First, we remark that the search criterion used by Joe and Sinescu [26, 46] is precisely $R_{N, s, \gamma}(z)$ for product weights, while the search criterion used in the other two papers mentioned above $[45,47]$ for general weights is

$$
\tilde{R}_{N, s, \gamma}(z):=\sum_{\emptyset \neq \mathfrak{u} \subseteq\{1: s\}} \gamma_{\mathfrak{u}}^{1 / 2} \tilde{R}_{N}\left(z_{\mathfrak{u}}\right),
$$

where

$$
\tilde{R}_{N}\left(\boldsymbol{z}_{\mathfrak{u}}\right):=\frac{1}{N} \sum_{k=0}^{N-1} \prod_{j \in \mathfrak{u}} \sum_{\substack{-N / 2<h \leq N / 2 \\ h \neq 0}} \frac{e^{2 \pi \mathrm{i} h k z_{j} / N}}{|h|} .
$$

(Remember that the weight $\gamma_{\mathfrak{u}}$ used by Joe and Sinescu [26, 46] should be replaced by $\gamma_{\mathfrak{u}}^{1 / 2}$ to be consistent with our notation here.) Notice that $\tilde{R}_{N}\left(z_{\mathfrak{u}}\right)$ in (4.13) differs from $R_{N}\left(z_{\mathfrak{l l}}\right)$ in (4.10) in two places: it does not have a 1 added to the sum over $h$, and it does not have the -1 at the end. It was proved by Sinescu and L'Ecuyer [47] for general $N \geq 2$ that the CBC construction based on $\tilde{R}_{N, s, \gamma}(z)$ yields

$$
\tilde{R}_{N, s, \gamma}(z) \leq \frac{2}{N} \sum_{\emptyset \neq \mathfrak{u} \subseteq\{1: s\}} \gamma_{\mathfrak{u}}^{1 / 2}\left(2 \ln N+\frac{2 \pi^{2}(N-1)}{3 \phi(N)}\right)^{|\mathfrak{|}|}
$$

and their proof did not require the monotonicity condition (4.9); the condition (4.9) was only used in the step for connecting the discrepancy $D_{s, \gamma}^{\infty, \infty}(\mathcal{P})$ to $\tilde{R}_{N, s, \gamma}(z)$.

In the proof of Theorem 4.1, we removed the constant $m$ from the product in the expression of $e_{N, s, \gamma}^{2}(z)$ by defining a set of auxiliary weights. Here we use the same argument to remove the constant 1 from the product in (4.10). We can show that

$$
R_{N, s, \gamma}(z)=\tilde{R}_{N, s, \tilde{\gamma}}(z)
$$

for some auxiliary weights

$$
\tilde{\gamma}_{\mathfrak{v}}^{1 / 2}:=\sum_{\mathfrak{v} \subseteq \mathfrak{u} \subseteq\{1: s\}} \gamma_{\mathfrak{u}}^{1 / 2}, \quad \mathfrak{v} \subseteq\{1: s\}
$$

Hence, the CBC construction based on $R_{N, s, \gamma}(z)$ is identical to the CBC construction based on $\tilde{R}_{N, s, \tilde{\gamma}}(z)$ for auxiliary weights $\tilde{\gamma}_{\mathfrak{v}}$, and the bound (4.14) applies, but with weights $\gamma_{\mathfrak{u}}$ replaced by $\tilde{\gamma}_{\mathfrak{v}}$. Upon writing $\alpha_{N}:=2 \ln N+\left(2 \pi^{2} / 3\right)(N-1) / \phi(N)$ we get

$$
\sum_{\mathfrak{\emptyset} \in \mathfrak{v} \subseteq\{1: s\}} \tilde{\gamma}_{\mathfrak{v}}^{1 / 2} \alpha_{N}^{|\mathfrak{p}|}=\sum_{\mathfrak{v} \subseteq\{1: s\}} \sum_{\mathfrak{v} \subseteq \mathfrak{u} \subseteq\{1: s\}} \gamma_{\mathfrak{u}}^{1 / 2} \alpha_{N}^{|\mathfrak{v}|}-\sum_{\mathfrak{u} \subseteq\{1: s\}} \gamma_{\mathfrak{u}}^{1 / 2}=\sum_{\emptyset \neq \mathfrak{u} \subseteq\{1: s\}} \gamma_{\mathfrak{u}}^{1 / 2}\left(1+\alpha_{N}\right)^{|\mathfrak{u}|} .
$$

This completes the proof. 
We can say more about the special case of product weights. We need the following result, which has appeared previously $[20,59]$ in a similar form. However, our choice of the parameters $a$ and $b$ here yields a better overall constant $a^{b}$.

Lemma 4.4. Suppose that $\tau_{j} \geq 0$ for all $j \geq 1$ and that $\sum_{j=1}^{\infty} \tau_{j}<\infty$. Let $\delta$, a and $b$ satisfy

$$
0<\delta<\min \left(1,2 \sum_{j=1}^{\infty} \tau_{j}\right), \quad a:=\frac{2}{\delta} \sum_{j=1}^{\infty} \tau_{j}, \quad \sum_{j=b+1}^{\infty} \tau_{j} \leq \frac{\delta}{2} .
$$

Then, for all $n>0$,

$$
\prod_{j=1}^{\infty}\left(1+\tau_{j} \ln n\right) \leq a^{b} n^{\delta}
$$

Proof. For arbitrary $a \geq 1$ and $b \geq 1$,

$$
\begin{aligned}
\prod_{j=1}^{\infty}\left(1+\tau_{j} \ln n\right) & \leq \prod_{j=1}^{b}\left(a+\tau_{j} \ln n\right) \prod_{j=b+1}^{\infty}\left(1+\tau_{j} \ln n\right) \\
& =a^{b} \prod_{j=1}^{b}\left(1+\frac{\tau_{j} \ln n}{a}\right) \prod_{j=b+1}^{\infty}\left(1+\tau_{j} \ln n\right) \\
& =a^{b} \exp \left(\sum_{j=1}^{b} \ln \left(1+\frac{\tau_{j} \ln n}{a}\right)+\sum_{j=b+1}^{\infty} \ln \left(1+\tau_{j} \ln n\right)\right) \\
& \leq a^{b} \exp \left(\frac{\ln n}{a} \sum_{j=1}^{b} \tau_{j}+\ln n \sum_{j=b+1}^{\infty} \tau_{j}\right)
\end{aligned}
$$

where we have used the property that $\ln (1+x) \leq x$ for all $x \geq 0$. The result follows by choosing $a$ and $b$ as specified.

Theorem 4.5. For any $\delta \in(0,1)$, if the weights satisfy

$$
\sum_{|\mathfrak{u}|<\infty} \gamma_{\mathfrak{u}}^{1 / 2}\left(\frac{c_{\text {lat }}|\mathfrak{u}|}{e \delta}\right)^{|\mathfrak{u}|}<\infty,
$$

or if we have product weights satisfying

$$
\sum_{j=1}^{\infty} \gamma_{j}^{1 / 2}<\infty
$$

then the lattice rule constructed by the CBC algorithm based on $R_{N, s, \gamma}(z)$ satisfies, for all $q^{\prime}, r^{\prime} \geq 1$,

$$
D_{s, \gamma}^{q^{\prime}, r^{\prime}}(\mathcal{P}) \leq C N^{-1+\delta},
$$

where $C$ is independent of $s$ and $N$ but depends on $\delta$ and tends to infinity as $\delta \rightarrow 0$. 
Proof. Sinescu and Joe [45] argue that Bernoulli's inequality yields $1-(1-1 / N)^{|\mathfrak{u}|} \leq$ $|\mathfrak{u}| / N$. Thus, for general weights, the first term on the right-hand side of (4.11) is bounded by

$$
\sum_{\emptyset \neq \mathfrak{u} \subseteq\{1: s\}} \gamma_{\mathfrak{u}}^{1 / 2}\left(1-\left(1-\frac{1}{N}\right)^{|\mathfrak{u}|}\right) \leq \frac{1}{N} \sum_{\emptyset \neq \mathfrak{u} \subseteq\{1: s\}} \gamma_{\mathfrak{u}}^{1 / 2}|\mathfrak{u}| .
$$

For the second term $R_{N, s, \gamma}(z) / 2$ on the right-hand side of (4.11), we use the bound in Theorem 4.3. It was shown by Sinescu and L'Ecuyer [47], and is easily verified, that $N^{-\delta}(\ln N)^{|\mathfrak{u}|} \leq(|\mathfrak{u}| /(e \delta))^{|\mathfrak{u}|}$, which yields

$$
R_{N, s, \gamma}(z) \leq \frac{2}{N^{1-\delta}} \sum_{\emptyset \neq \mathfrak{u} \subseteq\{1: s\}} \gamma_{\mathfrak{u}}^{1 / 2}\left(\frac{c_{\text {lat }}|\mathfrak{u}|}{e \delta}\right)^{|\mathfrak{u}|}
$$

The result then follows by combining these two estimates.

For product weights, it is proved by Joe [26] that

$$
\sum_{\mathfrak{u} \subseteq\{1: s\}} \gamma_{\mathfrak{u}}^{1 / 2}\left(1-\left(1-\frac{1}{N}\right)^{|\mathfrak{u}|}\right) \leq \frac{1}{N} \max \left(1, \sum_{j=1}^{\infty} \frac{\gamma_{j}^{1 / 2}}{1+\gamma_{j}^{1 / 2}}\right) \exp \left(\sum_{j=1}^{\infty} \gamma_{j}^{1 / 2}\right),
$$

while Theorem 4.3 yields $R_{N, s, \gamma}(z) \leq(2 / N) \prod_{j=1}^{s}\left(1+c_{\text {lat }} \gamma_{j}^{1 / 2} \ln N\right)$. We now use Lemma 4.4 to conclude that

$$
R_{N, s, \gamma}(z) \leq \frac{2}{N^{1-\delta}}\left(\frac{2 c_{\mathrm{lat}}}{\delta} \sum_{j=1}^{\infty} \gamma_{j}^{1 / 2}\right)^{b}
$$

where $b$ satisfies $c_{\text {lat }} \sum_{j=b+1}^{\infty} \gamma_{j}^{1 / 2} \leq \delta / 2$. The result for product weights then follows by combining these two estimates.

The following theorem allows us to construct a lattice rule with a lower convergence rate when the decay of weights is not sufficiently fast.

THEOREM 4.6. For any $\delta \in(0,1)$, suppose that we have general weights satisfying

$$
\sum_{|\mathfrak{u}|<\infty} \gamma_{\mathfrak{u}}^{v}\left(\frac{c_{\mathrm{lat}}|\mathfrak{u}|}{e \delta}\right)^{|\mathfrak{u}|}<\infty \quad \text { for some } v>\frac{1}{2}
$$

or product weights satisfying

$$
\sum_{j=1}^{\infty} \gamma_{j}^{v}<\infty \text { for some } v>\frac{1}{2}
$$

Define new weights $\tilde{\gamma}_{\mathfrak{u}}:=\gamma_{\mathfrak{u}}^{2 v}$ for all $|\mathfrak{u}|<\infty$ in the case of general weights, or $\tilde{\gamma}_{j}:=\gamma_{j}^{2 v}$ for all $j \geq 1$ in the case of product weights. Then the lattice rule constructed by the 
$C B C$ algorithm based on $R_{N, s, \tilde{\gamma}}(z)$ with new weights $\tilde{\gamma}$ satisfies, for all $q^{\prime} \geq 1$ and $r^{\prime} \geq 2 v$,

$$
D_{s, \gamma}^{q^{\prime}, r^{\prime}}(\mathcal{P}) \leq C N^{-1 /(2 v)+\delta}
$$

where $C$ is independent of $s$ and $N$ but depends on $v$ and $\delta$ and tends to infinity as $\delta \rightarrow 0$.

Proof. We have $\sum_{|\mathfrak{u}|<\infty} \tilde{\gamma}_{\mathfrak{u}}^{1 / 2}\left(c_{\text {lat }}|\mathfrak{u}| /(e \delta)\right)^{|\mathfrak{u}|}<\infty$ in the case of general weights, and $\sum_{j=1}^{\infty} \tilde{\gamma}_{j}^{1 / 2}<\infty$ in the case of product weights. Using Lemma 3.3 with $r_{2}^{\prime}=r^{\prime} \geq 2 v$ and $r_{1}^{\prime}=r^{\prime} /(2 v) \geq 1$, so that $r_{2}^{\prime} / r_{1}^{\prime}=2 v$, we obtain

$$
D_{s, \gamma}^{q^{\prime}, r^{\prime}}(\mathcal{P}) \leq\left[D_{s, \tilde{\gamma}}^{q^{\prime}, r^{\prime} /(2 v)}(\mathcal{P})\right]^{1 /(2 v)}
$$

The proof is completed by using this bound together with Theorem 4.5, with $\gamma$ replaced by $\tilde{\gamma}$.

We remark that the $\mathrm{CBC}$ constructions based on $e_{N, s, \gamma}^{2}(z)$ and $R_{N, s, \gamma}(z)$ can both be used in the Hilbert space setting with $q=r=2$. We now briefly discuss their pros and cons. Firstly, to obtain close to order $1 / N$ convergence in the case of product weights, both constructions require $\sum_{j=1}^{\infty} \gamma_{j}^{1 / 2}<\infty$; but if the weights satisfy a weaker condition, then a slower convergence rate is obtained. Similar results hold for general weights, but the required condition on general weights for $R_{N, s, \gamma}(z)$ appears to be tougher. Secondly, the quantity $e_{N, s, \gamma}^{2}(z)$ is some average of $D_{s, \gamma}^{2,2}(\mathcal{P})$ over random shifts, while $R_{N, s, \gamma}(z)$ is part of a very loose upper bound on $D_{s, \gamma}^{2,2}(\mathcal{P})$. Thus one might expect the quality of the lattice rule constructed from $e_{N, s, \gamma}^{2}(z)$ to be better. Finally, Theorem 4.2 is a probabilistic result and requires random shifts, while Theorems 4.5 and 4.6 are completely deterministic and do not require any shift (although shifts might still be used for practical error estimation).

4.3. Low-discrepancy sequences Niederreiter [35, 36], Halton [11] and Sobol' [55] sequences are low-discrepancy sequences in $[0,1]^{s}$ that can be generated explicitly and which are extensible in both $s$ and $N$. Furthermore, all projections of these sequences have good quality according to the classical star discrepancy bounds. Joe and Kuo [27] give parameters to construct Sobol' sequences in more than 12000 dimensions. Throughout this section, let $\mathcal{P}^{\mathrm{Nie}}, \mathcal{P}^{\mathrm{Hal}}$ and $\mathcal{P}^{\mathrm{Sob}}$ denote Niederreiter, Halton and Sobol' sequences, respectively.

Theorem 4.7. The first $N$ points of the $s$-dimensional sequence $\mathcal{P} \in\left\{\mathcal{P}^{\mathrm{Nie}}, \mathcal{P}^{\mathrm{Hal}}, \mathcal{P}^{\mathrm{Sob}}\right\}$ satisfy, for any $q^{\prime} \in[1, \infty)$ and $r^{\prime} \in[1, \infty)$,

$$
D_{s, \gamma}^{q^{\prime}, r^{\prime}}(\mathcal{P}) \leq D_{s, \gamma}^{\infty, r^{\prime}}(\mathcal{P})=\left(\sum_{\emptyset \neq \mathfrak{u} \subseteq\{1: s\}} \gamma_{\mathfrak{u}}^{r^{\prime} / 2}\left[D^{*}\left(\mathcal{P}_{\mathfrak{u}}\right)\right]^{r^{\prime}}\right)^{1 / r^{\prime}}
$$


with

$$
\begin{aligned}
D^{*}\left(\mathcal{P}_{\mathfrak{u}}^{\mathrm{Nie}}\right) & \leq \frac{1}{N} \prod_{j \in \mathfrak{u}}\left(c_{\mathrm{Nie}} j \log _{\kappa}(j+\kappa) \ln (\kappa N)\right), \\
D^{*}\left(\mathcal{P}_{\mathfrak{u}}^{\mathrm{Hal}}\right) & \leq \frac{1}{N} \prod_{j \in \mathfrak{u}}\left(c_{\mathrm{Hal}} j \log _{2}(j+1) \ln (e N)\right), \\
D^{*}\left(\mathcal{P}_{\mathfrak{u}}^{\mathrm{Sob}}\right) & \leq \frac{1}{N} \prod_{j \in \mathfrak{u}}\left(c_{\mathrm{Sob}} j \log _{2}(j+1) \log _{2} \log _{2}(j+3) \ln (2 N)\right),
\end{aligned}
$$

where $\kappa$ is the base for the Niederreiter sequence and $c_{\mathrm{Nie}}, c_{\mathrm{Hal}}$ and $c_{\mathrm{Sob}}$ are independent of $s$ and $N$. The $r^{\prime}=\infty$ case is obtained by the obvious adjustment.

Proof. The bounds on $D^{*}\left(\mathcal{P}_{\mathfrak{u}}^{\mathrm{Nie}}\right)$ and $D^{*}\left(\mathcal{P}_{\mathfrak{u}}^{\text {Sob }}\right)$ were proved by Wang [59], and the bound on $D^{*}\left(\mathcal{P}_{\mathfrak{u}}^{\mathrm{Hal}}\right)$ by Hickernell and Wang [20]. See also a paper of Sloan et al. [52].

Requirements on the weights are stated in the next theorem only for the simpler case of product weights. The theorem indicates that low-discrepancy sequences defined independently of weights can nevertheless adapt well to given weights. The conditions on the weights are stronger than (4.15) in Theorem 4.5, but it is not known whether or not the stronger requirements are artifacts of the method of proof.

Theorem 4.8. Let $\mathcal{P} \in\left\{\mathcal{P}^{\mathrm{Nie}}, \mathcal{P}^{\mathrm{Hal}}, \mathcal{P}^{\mathrm{Sob}}\right\}$. Suppose that for some $v \geq 1 / 2$ we have product weights satisfying

$$
\sum_{j=1}^{\infty} \gamma_{j}^{v} j \ln j<\infty \quad \text { when } \mathcal{P} \in\left\{\mathcal{P}^{\mathrm{Nie}}, \mathcal{P}^{\mathrm{Hal}}\right\},
$$

or

$$
\sum_{j=1}^{\infty} \gamma_{j}^{v} j \ln j \ln \ln j<\infty \quad \text { when } \mathcal{P}=\mathcal{P}^{\mathrm{Sob}} .
$$

Then the first $N$ points of the $s$-dimensional sequence $\mathcal{P}$ satisfy, for all $q^{\prime} \geq 1, r^{\prime} \geq 2 v$ and any $\delta>0$,

$$
D_{s, \gamma}^{q^{\prime}, r^{\prime}}(\mathcal{P}) \leq C N^{-1 /(2 v)+\delta},
$$

where $C$ is independent of $s$ and $N$ but depends on $v$ and $\delta$ and tends to infinity as $\delta \rightarrow 0$.

Proof. For product weights and using $D_{s, \gamma}^{q^{\prime}, r^{\prime}}(\mathcal{P}) \leq D_{s, \gamma}^{q^{\prime}, 1}(\mathcal{P})$, the discrepancy bounds in Theorem 4.7 lead to

$$
D_{s, \gamma}^{q^{\prime}, r^{\prime}}\left(\mathcal{P}^{\mathrm{Nie}}\right) \leq \frac{1}{N} \prod_{j=1}^{s}\left(1+\gamma_{j}^{1 / 2} c_{\mathrm{Nie}} j \log _{\kappa}(j+\kappa) \ln (\kappa N)\right),
$$




$$
\begin{aligned}
& D_{s, \gamma}^{q^{\prime}, r^{\prime}}\left(\mathcal{P}^{\mathrm{Hal}}\right) \leq \frac{1}{N} \prod_{j=1}^{s}\left(1+\gamma_{j}^{1 / 2} c_{\mathrm{Hal}} j \log _{2}(j+1) \ln (e N)\right), \\
& D_{s, \gamma}^{q^{\prime}, r^{\prime}}\left(\mathcal{P}^{\mathrm{Sob}}\right) \leq \frac{1}{N} \prod_{j=1}^{s}\left(1+\gamma_{j}^{1 / 2} c_{\mathrm{Sob}} j \log _{2}(j+1) \log _{2} \log _{2}(j+3) \ln (2 N)\right) .
\end{aligned}
$$

The result for $v=1 / 2$ then follows from Lemma 4.4. This result was proved by Wang [59] for $\mathcal{P}^{\mathrm{Nie}}$ and $\mathcal{P}^{\mathrm{Sob}}$, and by Hickernell and Wang [20] for $\mathcal{P}^{\mathrm{Hal}}$; in both papers the result was formulated for $D_{s, \gamma}^{2,2}(\mathcal{P})$.

To prove the result for $v>1 / 2$, we define new weights $\tilde{\gamma}_{j}:=\gamma_{j}^{2 v}$ for all $j \geq 1$. Using Lemma 3.3 as in the proof of Theorem 4.6, we obtain $D_{s, \gamma}^{q^{\prime}, r^{\prime}}(\mathcal{P}) \leq\left[D_{s, \tilde{\gamma}}^{q^{\prime}, r^{\prime} /(2 v)}(\mathcal{P})\right]^{1 /(2 v)}$. The proof is completed by inserting the bound for $D_{s, \tilde{\gamma}}^{q^{\prime}, r^{\prime} /(2 v)}(\mathcal{P})$.

\section{Fast CBC construction for POD weights}

The criteria used in Section 4 for the two lattice CBC constructions take similar forms: see (4.1) in the case of $e_{N, s, \gamma}^{2}(z)$ and (4.10) and (4.12) in the case of $R_{N, s, \gamma}(z)$. We now describe the fast $\mathrm{CBC}$ construction for the generic criterion

$$
e_{N, s}^{2}\left(z_{1}, \ldots, z_{s}\right):=\sum_{\emptyset \neq \mathfrak{u} \subseteq\{1: s\}} \gamma_{\mathfrak{u}}\left(\frac{1}{N} \sum_{k=0}^{N-1} \prod_{j \in \mathfrak{u}} \omega\left(\left\{\frac{k z_{j}}{N}\right\}\right)\right),
$$

in which the -1 and $-m^{|\mathfrak{u}|}$ terms have been omitted because they are independent of $z$, with POD weights; see (1.15). We need only very minor modifications of the "order dependent" case of Cools et al. [4, Section 4.1].

Suppose that we are at the point in the CBC algorithm where we want to choose the $d$ th component $z_{d}$. It makes sense to consider $e_{N, d}^{2}\left(z_{1}, \ldots, z_{d}\right)=: E_{d}^{2}\left(z_{d}\right)$ as a function of $z_{d}$. We can write

$$
\begin{aligned}
& E_{d}^{2}\left(z_{d}\right)= \frac{1}{N} \sum_{k=0}^{N-1} \sum_{\ell=1}^{d} \Gamma_{\ell} \sum_{\substack{\mathfrak{u} \subseteq\{1: d\} \\
|\mathfrak{u}|=\ell}} \prod_{j \in \mathfrak{u}}\left[\gamma_{j} \omega\left(\left\{\frac{k z_{j}}{N}\right\}\right)\right] \\
&= \frac{1}{N} \sum_{k=0}^{N-1} \sum_{\ell=1}^{d} \Gamma_{\ell}\left(\sum_{\substack{\mathfrak{u} \subseteq\{1: d-1\} \\
|\mathfrak{u}|=\ell}} \prod\left[\gamma_{j} \omega\left(\left\{\frac{k z_{j}}{N}\right\}\right)\right]\right. \\
&\left.+\gamma_{d} \omega\left(\left\{\frac{k z_{d}}{N}\right\}\right) \sum_{\substack{\mathfrak{u} \subseteq\{1: d-1\} \\
|\mathfrak{u}|=\ell-1}} \prod_{j \in \mathfrak{u}}\left[\gamma_{j} \omega\left(\left\{\frac{k z_{j}}{N}\right\}\right)\right]\right) \\
&=e_{N, d-1}^{2}\left(z_{1}, \ldots, z_{d-1}\right)+\frac{\gamma_{d}}{N} \sum_{k=0}^{N-1} \omega\left(\left\{\frac{k z_{d}}{N}\right\}\right)\left(\sum_{\ell=1}^{d} \Gamma_{\ell} p_{d-1, \ell-1}(k)\right)
\end{aligned}
$$


where $e_{N, 0}^{2}:=0$ and the products $p_{d, \ell}(k)$ are defined recursively by

$$
\begin{aligned}
& p_{d, 0}(k):=1 \\
& p_{d, \ell}(k):=p_{d-1, \ell}(k)+\gamma_{d} \omega\left(\left\{\frac{k z_{d}}{N}\right\}\right) p_{d-1, \ell-1}(k) .
\end{aligned}
$$

Let $\mathbb{Z}_{N}:=\{0,1, \ldots, N-1\}$ denote the set of integers modulo $N$, and let $U_{N}:=\{u \in$ $\left.\mathbb{Z}_{N}: \operatorname{gcd}(u, N)=1\right\}$ denote the multiplicative group of integers modulo $N$ as before, with $\left|U_{N}\right|=\phi(N)$. We need to evaluate $E_{d}^{2}\left(z_{d}\right)$ for every choice of $z_{d} \in U_{N}$, which suggests the definition of the vectors

$$
\boldsymbol{E}_{d}^{2}:=\left[E_{d}^{2}(z)\right]_{z \in U_{N}}, \quad \boldsymbol{p}_{d, \ell}:=\left[p_{d, \ell}(k)\right]_{k \in \mathbb{Z}_{N}}
$$

and the matrix

$$
\mathbf{\Omega}_{N}:=\left[\omega\left(\left\{\frac{k z}{N}\right\}\right)\right]_{\substack{z \in U_{N} \\ k \in \mathbb{Z}_{N}}}=\left[\omega\left(\frac{k z \bmod N}{N}\right)\right]_{\substack{z \in U_{N} \\ k \in \mathbb{Z}_{N}}} .
$$

We now observe from (5.1) that the vector $\boldsymbol{E}_{d}^{2}$ can be expressed in terms of a matrixvector product, involving the matrix $\boldsymbol{\Omega}_{N}$, as

$$
\boldsymbol{E}_{d}^{2}:=\mathbf{1}_{\phi(N)} e_{N, d-1}^{2}\left(z_{1}, \ldots, z_{d-1}\right)+\frac{\gamma_{d}}{N} \boldsymbol{\Omega}_{N}\left(\sum_{\ell=1}^{d} \Gamma_{\ell} \boldsymbol{p}_{d-1, \ell-1}\right)
$$

where $\mathbf{1}_{t}$ denotes a vector of ones of length $t$. The CBC algorithm picks the value of $z_{d} \in U_{N}$ that corresponds to the smallest entry in $\boldsymbol{E}_{d}^{2}$. Then it is clear from (5.2) that the vectors $\boldsymbol{p}_{d, \ell}$ for the next iteration can be obtained recursively via

$$
\boldsymbol{p}_{d, \ell}:=\boldsymbol{p}_{d-1, \ell}+\boldsymbol{\Omega}_{N}\left(z_{d}\right) * \boldsymbol{p}_{d-1, \ell-1},
$$

where $\boldsymbol{\Omega}_{N}\left(z_{d}\right)$ denotes the row of $\boldsymbol{\Omega}_{N}$ corresponding to the chosen $z_{d}$, and the operator * denotes elementwise vector multiplication. Since the vectors $\boldsymbol{p}_{d-1, \ell}$ are no longer needed in the next iteration, we can simply overwrite $\boldsymbol{p}_{d-1, \ell}$ with $\boldsymbol{p}_{d, \ell}$. Hence, starting with the vectors $\boldsymbol{p}_{0, \ell}:=\mathbf{1}_{N}$, we require only $\mathrm{O}(s N)$ storage for POD weights.

The trick now is to order the indices $z \in U_{N}$ and $k \in \mathbb{Z}_{N}$ in (5.3) and (5.4) in a clever way so as to allow fast matrix-vector multiplications. For this, we can follow the discussion of Cools et al. [4, Section 4.2], which covers prime $N$ and the case where $N$ is a power of a prime. In particular, the only change needed specifically for POD weights is the update step [4, top of p. 2177],

$$
\boldsymbol{p}_{d, \ell}^{\left\langle g^{-1}\right\rangle}:=\Pi_{g^{-1}}^{\top} \boldsymbol{p}_{d, \ell}=\boldsymbol{p}_{d-1, \ell}^{\left\langle g^{-1}\right\rangle}+\gamma_{d} \mathbf{\Omega}_{p^{m}}^{\left\langle g^{-1}\right\rangle}\left(z_{d}\right) . * \boldsymbol{p}_{d-1, \ell-1}^{\left\langle g^{-1}\right\rangle}
$$

The overall CBC construction cost is then $\mathrm{O}(s N \ln N)$ operations. 


\section{Acknowledgements}

The authors thank Henryk Woźniakowski for valuable suggestions. Frances Kuo was supported by an Australian Research Council QEII Fellowship, an Australian Research Council Discovery Project, and the Vice-Chancellor's Childcare Support Fund for Women Researchers at the University of New South Wales. Christoph Schwab was supported by the Swiss National Science Foundation under grant no. 200021-120290/1, and by the European Research Council under FP7 grant AdG247277. Ian Sloan was supported by Australian Research Council Discovery and Centre of Excellence grants.

\section{References}

[1] N. Aronszajn, "Theory of reproducing kernels", Trans. Amer. Math. Soc. 68 (1950) 337-404; doi:10.2307/1990404.

[2] R. Bellman, Dynamic programming (Princeton University Press, Princeton, 1957).

[3] R. E. Caflisch, W. Morokoff and A. Owen, "Valuation of mortgage backed securities using Brownian bridges to reduce effective dimension", J. Comput. Finance 1 (1997) 27-46.

[4] R. Cools, F. Y. Kuo and D. Nuyens, "Constructing embedded lattice rules for multivariate integration", SIAM J. Sci. Comput. 28 (2006) 2162-2188; doi:10.1137/06065074X.

[5] J. Dick, "On the convergence rate of the component-by-component construction of good lattice rules", J. Complexity 20 (2004) 493-522; doi:10.1016/j.jco.2003.11.008.

[6] J. Dick and F. Pillichshammer, Digital nets and sequences (Cambridge University Press, Cambridge, 2010).

[7] J. Dick, F. Pillichshammer and B. J. Waterhouse, "The construction of good extensible rank-1 lattices", Math. Comp. 77 (2008) 2345-2374; doi:10.1090/S0025-5718-08-02009-7.

[8] H. Faure, "Discrépance de suites associées à un système de numération (en dimension s)", Acta Arith. 41 (1982) 337-351.

[9] M. Giles, F. Y. Kuo, I. H. Sloan and B. J. Waterhouse, "Quasi-Monte Carlo for finance applications", ANZIAM J. 50 (2008) C308-C323 (Proc. CTAC 2008).

[10] M. Gnewuch, "Infinite-dimensional integration on weighted Hilbert spaces", Math. Comp. in press; doi:10.1090/S0025-5718-2012-02583-X.

[11] J. H. Halton, "On the efficiency of certain quasi-random sequences of points in evaluating multidimensional integrals", Numer. Math. 2 (1960) 84-90; doi:10.1007/BF01386213.

[12] S. Heinrich, H. Woźniakowski, G. W. Wasilkowski and E. Novak, "The inverse of the stardiscrepancy depends linearly on the dimension", Acta Arith. 96 (2001) 279-302; doi:10.4064/aa96-3-7.

[13] F. J. Hickernell, "A generalized discrepancy and quadrature error bound", Math. Comp. 67 (1998) 299-322; doi:10.1090/S0025-5718-98-00894-1.

[14] F. J. Hickernell, "Lattice rules: How well do they measure up?", in: Random and quasi-random point sets (eds P. Hellekalek and G. Larcher), (Springer, Berlin, 1998) 109-166.

[15] F. J. Hickernell, T. Müller-Gronbach, B. Niu and K. Ritter, "Multi-level Monte Carlo algorithms for infinite-dimensional integration on $\mathbb{R}^{\mathbb{N}}$, J. Complexity 26 (2010) 229-254; doi:10.1016/j.jco.2010.02.002.

[16] F. J. Hickernell, I. H. Sloan and G. W. Wasilkowski, "On strong tractability of weighted multivariate integration", Math. Comp. 73 (2004) 1903-1911; doi:10.1090/S0025-5718-04-01653-9.

[17] F. J. Hickernell, I. H. Sloan and G. W. Wasilkowski, "On tractability of weighted integration for certain Banach spaces of functions", in: Monte Carlo and quasi-Monte Carlo methods 2002 (ed. H. Niederreiter), (Springer, Berlin, 2004) 51-71. 
[18] F. J. Hickernell, I. H. Sloan and G. W. Wasilkowski, "On tractability of weighted integration over bounded and unbounded regions in $\mathbb{R}^{s ",}$, Math. Comp. 73 (2004) 1885-1901; doi:10.1090/S0025-5718-04-01624-2.

[19] F. J. Hickernell, I. H. Sloan and G. W. Wasilkowski, "The strong tractability of multivariate integration using lattice rules", in: Monte Carlo and quasi-Monte Carlo methods 2002 (ed. H. Niederreiter), (Springer, Berlin, 2004) 259-273.

[20] F. J. Hickernell and X. Wang, "The error bounds and tractability of quasi-Monte Carlo algorithms in infinite dimension", Math. Comp. 71 (2002) 1641-1661; doi:10.1090/S0025-5718-01-01377-1.

[21] F. J. Hickernell and H. Woźniakowski, "Integration and approximation in arbitrary dimensions", Adv. Comput. Math. 12 (2000) 25-58; doi:10.1023/A:1018948631251.

[22] A. Hinrichs, F. Pillichshammer and W. Ch. Schmid, "Tractability properties of the weighted star discrepancy", J. Complexity 24 (2008) 134-143; doi:10.1016/j.jco.2007.08.002.

[23] E. Hlawka, "Funktionen von beschränkter Variation in der Theorie der Gleichverteilung", Ann. Mat. Pura Appl. (4) 54 (1961) 325-333; doi:10.1007/BF02415361.

[24] E. Hlawka, "Über die Diskrepanz mehrdimensionaler Folgen mod. 1", Math. Z. 77 (1961) 273-284; doi:10.1007/BF01180179.

[25] S. Joe, "Formulas for the computation of the weighted $L^{2}$ discrepancy", Research Report No. 55, Department of Mathematics, University of Waikato, 1997.

[26] S. Joe, "Construction of good rank-1 lattice rules based on the weighted star discrepancy", in: Monte Carlo and quasi-Monte Carlo methods 2004 (eds H. Niederreiter and D. Talay), (Springer, Berlin, 2006) 181-196.

[27] S. Joe and F. Y. Kuo, "Constructing Sobol' sequences with better two-dimensional projections", SIAM J. Sci. Comput. 30 (2008) 2635-2654; doi:10.1137/070709359.

[28] J. F. Koksma, "Een algemeene stelling uit de theorie der gelijkmatige verdeeling modulo 1", Mathematica B (Zutphen) 11 (1942/43) 7-11.

[29] N. M. Korobov, Teoretiko-chislovye metody v priblizhennom analize (Gosudarstv. Izdat. Fiz. Mat. Lit., Moscow, 1963) Russian (Number-theoretic methods in approximate analysis).

[30] F. Y. Kuo, "Component-by-component constructions achieve the optimal rate of convergence in weighted Korobov and Sobolev spaces", J. Complexity 19 (2003) 301-320; doi:10.1016/S0885-064X(03)00006-2.

[31] F. Y. Kuo, Ch. Schwab and I. H. Sloan, "Quasi-Monte Carlo finite element methods for a class of elliptic partial differential equations with random coefficients", Research Report 2011-52, Seminar for Applied Mathematics, ETH Zürich, 2011, http://www.sam.math.ethz.ch/reports/2011/52.

[32] F. Y. Kuo, I. H. Sloan, G. W. Wasilkowski and B. J. Waterhouse, "Randomly shifted lattice rules with the optimal rate of convergence for unbounded integrands", J. Complexity 26 (2010) 135-160; doi:10.1016/j.jco.2009.07.005.

[33] F. Y. Kuo, I. H. Sloan, G. W. Wasilkowski and H. Woźniakowski, "On decompositions of multivariate functions", Math. Comp. 79 (2010) 953-966; doi:10.1090/S0025-5718-09-02319-9.

[34] F. Y. Kuo, I. H. Sloan, G. W. Wasilkowski and H. Woźniakowski, "Liberating the dimension", J. Complexity 26 (2010) 422-454; doi:10.1016/j.jco.2009.12.003.

[35] H. Niederreiter, "Low-discrepancy and low-dispersion sequences", J. Number Theory 30 (1988) 51-70; doi:10.1016/0022-314X(88)90025-X.

[36] H. Niederreiter, Random number generation and quasi-Monte Carlo methods (SIAM, Philadelphia, 1992).

[37] H. Niederreiter and C. Xing, "Low-discrepancy sequences and global function fields with many rational places", Finite Fields Appl. 2 (1996) 241-273; doi:10.1006/ffta.1996.0016.

[38] B. Niu, F. J. Hickernell, T. Müller-Gronbach and K. Ritter, "Deterministic multi-level algorithms for infinite-dimensional integration on $\mathbb{R}^{\mathbb{N}}$, J. Complexity 27 (2011) 331-351; doi:10.1016/j.jco.2010.08.001.

[39] E. Novak and H. Woźniakowski, "Intractability results for integration and discrepancy", J. Complexity 17 (2001) 388-441; doi:10.1006/jcom.2000.0577. 
[40] E. Novak and H. Woźniakowski, Tractability of multivariate problems. Volume I: Linear information, Volume 6 of EMS Tracts in Mathematics (European Mathematical Society, Zürich, 2008).

[41] E. Novak and H. Woźniakowski, Tractability of multivariate problems. Volume II: Standard information for functionals, Volume 12 of EMS Tracts in Mathematics (European Mathematical Society, Zürich, 2010).

[42] D. Nuyens and R. Cools, "Fast algorithms for component-by-component construction of rank 1 lattice rules in shift-invariant reproducing kernel Hilbert spaces", Math. Comp. 75 (2006) 903-920; doi:10.1090/S0025-5718-06-01785-6.

[43] D. Nuyens and R. Cools, "Fast component-by-component construction of rank-1 lattice rules with a nonprime number of points", J. Complexity 22 (2006) 4-28; doi:10.1016/j.jco.2005.07.002.

[44] L. Plaskota and G. W. Wasilkowski, "Tractability of infinite-dimensional integration in the worst case and randomized settings", J. Complexity 27 (2001) 505-518; doi:10.1016/j.jco.2011.01.006.

[45] V. Sinescu and S. Joe, "Good lattice rules based on the general weighted star discrepancy", Math. Comp. 76 (2007) 989-1004; doi:10.1090/S0025-5718-06-01943-0.

[46] V. Sinescu and S. Joe, "Good lattice rules with a composite number of points based on the product weighted star discrepancy", in: Monte Carlo and quasi-Monte Carlo methods 2006 (eds A. Keller, S. Heinrich and H. Niederreiter), (Springer, Berlin, 2008) 645-658.

[47] V. Sinescu and P. L'Ecuyer, "Existence and construction of shifted lattice rules with an arbitrary number of points and bounded weighted star discrepancy for general decreasing weights", J. Complexity 27 (2011) 449-465; doi:10.1016/j.jco.2011.02.001.

[48] I. H. Sloan and S. Joe, Lattice methods for multiple integration (Oxford University Press, Oxford, 1994).

[49] I. H. Sloan, F. Y. Kuo and S. Joe, "On the step-by-step construction of quasi-Monte Carlo integration rules that achieve strong tractability error bounds in weighted Sobolev spaces", Math. Comp. 71 (2002) 1609-1640; doi:10.1090/S0025-5718-02-01420-5.

[50] I. H. Sloan, F. Y. Kuo and S. Joe, "Constructing randomly shifted lattice rules in weighted Sobolev spaces”, SIAM J. Numer. Anal. 40 (2002) 1650-1665; doi:10.1137/S0036142901393942.

[51] I. H. Sloan and A. V. Reztsov, "Component-by-component construction of good lattice rules", Math. Comp. 71 (2002) 263-273; doi:10.1090/S0025-5718-01-01342-4.

[52] I. H. Sloan, X. Wang and H. Woźniakowski, "Finite-order weights imply tractability of multivariate integration”, J. Complexity 20 (2004) 46-74; doi:10.1016/j.jco.2003.11.003.

[53] I. H. Sloan and H. Woźniakowski, "When are quasi-Monte Carlo algorithms efficient for highdimensional integrals?”, J. Complexity 14 (1998) 1-33; doi:10.1006/jcom.1997.0463.

[54] I. H. Sloan and H. Woźniakowski, "Tractability of multivariate integration for weighted Korobov classes", J. Complexity 17 (2001) 697-721; doi:10.1006/jcom.2001.0599.

[55] I. M. Sobol', "On the distribution of points in a cube and the approximate evaluation of integrals", Zh. Vȳchisl. Mat. Mat. Fiz. 7 (1967) 784-802. English translation: U.S.S.R. Comput. Math. Math. Phys. 7 (1967) 86-112; doi:10.1016/0041-5553(67)90144-9.

[56] I. M. Sobol', Multidimensional quadrature formulas and Haar functions (Nauka, Moscow, 1969).

[57] I. M. Sobol', "Sensitivity estimates for nonlinear mathematical models", Matematicheskoe Modelirovanie 2 (1990) 112-118; English translation: Math. Model. Comput. Experiment 1 (1993) 407-414.

[58] A. H. Stroud, Approximate calculation of multiple integrals (Prentice-Hall, Englewood Cliffs, NJ, 1971).

[59] X. Wang, "Strong tractability of multivariate integration using quasi-Monte Carlo algorithms", Math. Comp. 72 (2003) 823-838; doi:10.1090/S0025-5718-02-01440-0.

[60] T. T. Warnock, "Computational investigations of low-discrepancy point sets", in: Applications of number theory to numerical analysis (ed. S. K. Zaremba), (Academic Press, New York, 1972) 319-343.

[61] S. K. Zaremba, "Some applications of multidimensional integration by parts", Ann. Polon. Math. 21 (1968) 85-96. 